Tôhoku Math. Journ.

31 (1979), 319-347.

\title{
COMPACTIFICATIONS OF THE MODULI SPACES OF HYPERELLIPTIC SURFACES
}

\author{
HiRoyasu TSUCHIHASHI
}

(Received September 18, 1978)

Hyperelliptic surfaces are classified into seven types [1]. In this paper we aim at constructing the moduli space of each type in the sense described in Mumford [8] and its compactification in some sense.

In Section 1, we study the structure of hyperelliptic surfaces and, by considering hyperelliptic surfaces with some base points, we get the fine moduli space $M_{i}$ of each type as a quotient space of the upper half plane or the product of two copies of the upper half plane, over which Suwa [11] showed the existence of a family of hyperelliptic surfaces complete and effectively parametrized at each point.

In Section 2, we construct the compactification $\bar{M}_{i}$ of $M_{i}$. And as a preparation for Section 3, we describe the resolution of certain quotient singularities in terms of torus embeddings.

In Section 3, we describe "degenerate hyperelliptic surfaces" represented by the boundary points of $\bar{M}_{i}$.

1. We write the elements of $C^{2}$ and $Z^{4}$ as row vectors. Let $\Omega$ be a $2 \times 2$ matrix with coefficients in $C$ of which the imaginary part is positive definite $\operatorname{Im}(\Omega)>0$. Then by $A(\Omega)$ we denote the complex torus of dimension 2 with the period matrix $\Omega$, i.e.,

$$
A(\Omega)=C^{2} / Z^{4}\left(\begin{array}{c}
\Omega \\
I
\end{array}\right) \text {. }
$$

Let $[x, y]$ denote the point of $A(\Omega)$ which is the image of $(x, y)$ in $C^{2}$.

We denote the upper half plane by $\mathfrak{S}$, and for any element $\tau$ of $\mathfrak{K}$, we denote by $E(\tau)$ the elliptic curve with the periods 1 and $\tau$, i.e.,

$$
E(\tau)=C /(\boldsymbol{Z} \tau+\boldsymbol{Z}) \text {. }
$$

We identify the elliptic curve $E$ with its group $\operatorname{Aut}(E)^{\circ}$ of translations, and let $[x]$ denote the point of $E(\tau)$ which is the image of $x$ in $C$.

Definition. By a hyperelliptic surface we mean an elliptic bundle over an elliptic curve whose total space has the first Betti number $b_{1}=2$.

THEOREM 1 [11]. Hyperelliptic surfaces are topologically classified 
into seven types, and any hyperelliptic surface can be expressed as the quotient space of an abelian surface $A$ by the group generated by an automorphism $g$ of $A$ as follows, where $\sigma_{N}=\exp (2 \pi i / N)$ and $\tau, \omega \in \mathfrak{S}$ :

$$
\begin{aligned}
& A=A\left(\left(\begin{array}{cc}
\tau & 0 \\
0 & \omega
\end{array}\right)\right) \\
& g:[x, y] \mapsto[x+1 / 2,-y] \\
& A=A\left(\left(\begin{array}{cc}
\tau & 1 / 2 \\
0 & \omega
\end{array}\right)\right) \\
& A=A\left(\left(\begin{array}{cc}
\tau & 0 \\
0 & \sigma_{3}
\end{array}\right)\right) \\
& A=A\left(\left(\begin{array}{cc}
\tau & \left(1-\sigma_{3}\right) / 3 \\
0 & \sigma_{3}
\end{array}\right)\right) \\
& A=A\left(\left(\begin{array}{cc}
\tau & 0 \\
0 & \sigma_{4}
\end{array}\right)\right) \\
& g:[x, y] \mapsto\left[x+1 / 4, \sigma_{4} y\right] \\
& A=A\left(\left(\begin{array}{cc}
\tau & \left(1+\sigma_{4}\right) / 2 \\
0 & \sigma_{4}
\end{array}\right)\right) \\
& A=A\left(\left(\begin{array}{cc}
\tau & 0 \\
0 & \sigma_{3}
\end{array}\right)\right) \\
& g:[x, y] \mapsto\left[x+1 / 3, \sigma_{3} y\right]
\end{aligned}
$$

We denote these surfaces by $S_{1}(\tau, \omega), S_{2}(\tau, \omega), S_{3}(\tau), S_{4}(\tau), S_{5}(\tau), S_{6}(\tau)$ and $S_{7}(\tau)$, respectively, and denote the point of $S_{i}(\tau, \omega)$ or $S_{i}(\tau)$ which is the image of $[x, y]$ of $A(\Omega)$ by the same notation $[x, y]$.

REMARK 1. $\tilde{\mathscr{S}}_{1}=\left\{S_{1}(\tau, \omega) \mid(\tau, \omega) \in \mathscr{S}^{2}\right\}$ form an analytic family effectively parametrized and complete at each point of $\mathfrak{S}^{2}$. In fact, we can construct this as follows: Let

$$
\mathscr{S}_{1}=\boldsymbol{C}^{2} \times \mathscr{S}^{2} /\left\{f_{a} \mid a \in Z^{4}\right\}
$$

with

$$
f_{a}:(x, y, \tau, \omega) \mapsto(x, y, \tau, \omega)+a\left(\begin{array}{cccc}
\tau & 0 & 0 & 0 \\
0 & \omega & 0 & 0 \\
1 & 0 & 0 & 0 \\
0 & 1 & 0 & 0
\end{array}\right)
$$

and

$$
\tilde{\mathscr{S}_{1}}=\tilde{\mathscr{A}} \tilde{\mathbb{I}}_{1} \quad \mathrm{~g}:[x, y, \tau, \omega] \mapsto[x+1 / 2,-y, \tau, \omega],
$$

where $[x, y, \tau, \omega]$ denotes the point of $\tilde{\mathscr{S}}_{1}$ which is the image of $(x, y$, $\tau, \omega)$ in $C^{2} \times \mathscr{S}^{2}$. Then $\tilde{\mathscr{S}}_{1}$ is non-singular, the holomorphic map $\tilde{\pi}_{1}: \tilde{\mathscr{S}}_{1} \rightarrow$ 
$\mathfrak{S}^{2}$ induced by the projection $C^{2} \times \mathscr{S}^{2} \rightarrow \mathscr{S}^{2}$ is smooth and $\tilde{\pi}_{1}^{-1}(\tau, \omega) \cong S_{1}(\tau, \omega)$. In the other cases, we can also construct the analytic families $\tilde{\pi}_{2}: \tilde{\mathscr{S}}_{2} \rightarrow \mathscr{S}^{2}$ and $\tilde{\pi}_{i}: \tilde{\mathscr{S}}_{i} \rightarrow \mathscr{S} \quad i=3,4,5,6,7$ in the same way as above.

Definition. $\quad \Gamma=S L(2, Z) /\{ \pm I\}$

$$
\begin{aligned}
& \Gamma_{o}(N)=\left\{\left(\begin{array}{ll}
a & b \\
c & d
\end{array}\right) \in S L(2, \boldsymbol{Z}) \mid c \equiv 0(\bmod N)\right\} /\{ \pm I\} \\
& \bar{\Gamma}_{o}(N)=\left\{\left(\begin{array}{ll}
a & b \\
c & d
\end{array}\right) \in S L(2, \boldsymbol{Z}) \mid a, d \equiv 1, c \equiv 0(\bmod N)\right\} \\
& \text { for } N=3,4,6 \\
& \bar{\Gamma}_{\circ}(8)=\left\{\left(\begin{array}{ll}
a & b \\
c & d
\end{array}\right) \in S L(2, Z) \mid a, d \equiv 1 \quad(\bmod 4), \quad c \equiv 0 \quad(\bmod 8)\right\} \\
& \bar{\Gamma}_{\circ}(9)=\left\{\left(\begin{array}{ll}
a & b \\
c & d
\end{array}\right) \in S L(2, Z) \mid a, d \equiv 1 \quad(\bmod 3), \quad c \equiv 0 \quad(\bmod 9)\right\} .
\end{aligned}
$$

REMARK 2. When $N=3,4,6,8$ or 9 , the projection $S L(2, Z) \rightarrow \Gamma$ obviously induces an isomorphism $\bar{\Gamma}_{o}(N) \rightarrow \Gamma_{o}(N)$. [2]).

On the other hand, the following is well known. (See, for instance,

Proposition 1. $\mathfrak{S} / \Gamma$ and $\mathfrak{E} / \Gamma_{\circ}(N)$ are punctured Riemann surfaces. Especially when $N=2,3,4,6,8$ or $9, \mathfrak{S}_{2} / \Gamma_{o}(N)$ is of genus 0 with $t=$ $2,2,3,4,4$ or 4 points removed, respectively.

Proposition 2. Let

$$
\begin{aligned}
& M_{1}^{\prime}=\mathfrak{S}_{\mathfrak{C}} / \Gamma_{\mathrm{o}}(2) \times \mathfrak{F} / \Gamma, \quad M_{2}^{\prime}=\left(\mathfrak{Y} / \Gamma_{\mathrm{o}}(2)\right)^{2}, \\
& M_{3}^{\prime}=M_{4}^{\prime}=\mathfrak{S}_{\mathcal{E}} / \Gamma_{0}(3), \quad M_{5}^{\prime}=M_{6}^{\prime}=\mathfrak{S}_{\mathfrak{C}} / \Gamma_{\mathrm{o}}(4), \quad M_{7}^{\prime}=\mathfrak{F}_{\mathcal{C}} / \Gamma_{\mathrm{o}}(6) \text {. }
\end{aligned}
$$

Then $M_{i}^{\prime}$ is the space of isomorphism classes of hyperelliptic surfaces of type (i).

REMARK 3. We can show as in the proof of Theorem 2 below that $M_{i}^{\prime}$ is in fact the coarse moduli space for hyperelliptic surfaces of type (i) in the sense of Mumford [8].

Proof of Proposition 2. For any hyperelliptic surface $S$, an abelian surface $A$ such that $S=A / g^{z}$ as in Theorem 1 , is uniquely determined by $S$. Indeed, $A$ is determined as the unramified covering manifold of $S$ of degree $m$ on which the pull back of the canonical bundle $K_{S}$ of $S$ is trivial, where $m$ is the order of $K_{S}$ in $\operatorname{Pic}(S)$. In this case $g^{z}$ is the covering transformation group and the only elements 
which can generate $g^{Z}$ are $g$ and $g^{-1}$. Hence if we set $S=A(\Omega) / g^{Z}$ and $S^{\prime}=A\left(\Omega^{\prime}\right) /\left(g^{\prime}\right)^{Z}$, then $S$ and $S^{\prime}$ are isomorphic if and only if there exists an isomorphism of complex manifolds $\varphi: A\left(\Omega^{\prime}\right) \rightarrow A(\Omega)$ such that $\varphi \circ g^{\prime}=$ $g \circ \varphi$ or $\phi \circ g^{\prime}=g^{-1} \circ \varphi$. The following facts are straightforward.

LEMMA. $A(\Omega)$ and $A\left(\Omega^{\prime}\right)$ are isomorphic as complex manifolds if and only if there exists a matrix

$$
\left(\begin{array}{ll}
A & B \\
C & D
\end{array}\right) \in G L(4, Z)
$$

such that $\Omega^{\prime}=(A \Omega+B)(C \Omega+D)^{-1}$. In this case, the isomorphism $\varphi: A\left(\Omega^{\prime}\right) \rightarrow A(\Omega)$ is induced by the affine transformation

$$
\widetilde{\varphi}:(x, y) \mapsto(x, y)(C \Omega+D)+(\alpha, \beta)
$$

of $C^{2}$ for some $(\alpha, \beta) \in C^{2}$.

On the other hand, $g$ is induced by the affine transformation

$$
\tilde{g}:(x, y) \mapsto(x, y)\left(\begin{array}{rr}
1 & 0 \\
0 & \sigma_{N}^{\prime}
\end{array}\right)+(1 / N, 0)
$$

of $C^{2}$, where $\sigma_{N}^{\prime}=\sigma_{N}$ when $N=2,3,4$ and $\sigma_{6}^{\prime}=-\sigma_{3}$. Thus we have an equality

$$
\left\{\varphi \circ g^{\prime}-g^{ \pm 1} \circ \varphi\right\}[x, y]=\left[\left\{\widetilde{\varphi} \circ \widetilde{g}^{\prime}-\widetilde{g}^{ \pm 1} \circ \widetilde{\varphi}\right\}(x, y)\right] .
$$

Hence $\varphi \circ g^{\prime}=g^{ \pm 1} \circ \varphi$ if and only if

$$
\left\{\widetilde{\varphi} \circ \widetilde{g}^{\prime}-\widetilde{g}^{ \pm 1} \circ \widetilde{\varphi}\right\}(x, y) \in Z^{4}\left(\begin{array}{l}
\Omega \\
I
\end{array}\right)
$$

for any $(x, y) \in C^{2}$. When this condition is satisfied, we see by easy calculation that $A, B, C$ and $D$ are diagonal matrices in the case of types (1), (3), (5), (7) or triangular matrices of which the $(2,1)$-entry is 0 in the case of types (2), (4), (6). Let the $(1,1)$-entry of $A, B, C$ and $D$ be $a_{1}, b_{1}, c_{1}$ and $d_{1}$, respectively, the (2,2)-entry of $A, B, C$ and $D$ be $a_{2}, b_{2}$, $c_{2}$ and $d_{2}$, respectively, and

$$
\Omega=\left(\begin{array}{cc}
\tau & * \\
0 & \omega
\end{array}\right) \quad \Omega^{\prime}=\left(\begin{array}{cc}
\tau^{\prime} & * \\
0 & \omega^{\prime}
\end{array}\right) .
$$

Then from $\Omega^{\prime}=(A \Omega+B)(C \Omega+D)^{-1}$, we get

$$
\tau^{\prime}=\frac{a_{1} \tau+b_{1}}{c_{1} \tau+d_{1}}, \quad \omega^{\prime}=\frac{a_{2} \omega+b_{2}}{c_{2}} \omega+d_{2},
$$

and 


$$
\operatorname{det}\left(\begin{array}{ll}
a_{1} & b_{1} \\
c_{1} & d_{1}
\end{array}\right)=\operatorname{det}\left(\begin{array}{ll}
a_{2} & b_{2} \\
c_{2} & d_{2}
\end{array}\right)=1
$$

Moreover from $\left\{\widetilde{\varphi} \circ \widetilde{g}^{\prime}-\widetilde{g}^{ \pm 1} \circ \widetilde{\varphi}\right\}(x, y) \in Z^{4}\left(\begin{array}{c}\Omega \\ I\end{array}\right)$, we get $c_{1} \equiv 0(\bmod N)$ and particularly in the case of type $(2) c_{2} \equiv 0(\bmod 2)$.

Conversely, assume that there exist $\gamma$ and $\delta$ such that $\tau^{\prime}=\gamma(\tau)$ and $\omega^{\prime}=\delta(\omega)$, and let

$$
\gamma=\left[\begin{array}{ll}
a_{1} & b_{1} \\
c_{1} & d_{1}
\end{array}\right], \delta=\left[\begin{array}{ll}
a_{2} & b_{2} \\
c_{2} & d_{2}
\end{array}\right] \quad\left(\begin{array}{ll}
a_{1} & b_{1} \\
c_{1} & d_{1}
\end{array}\right),\left(\begin{array}{ll}
a_{2} & b_{2} \\
c_{2} & d_{2}
\end{array}\right) \in \bar{\Gamma}_{o}(N) .
$$

Then we have

$$
\begin{gathered}
\Omega^{\prime}=(A \Omega+B)(C \Omega+D)^{-1}, \quad\left(\begin{array}{ll}
A & B \\
C & D
\end{array}\right) \in G L(4, Z) \\
\left\{\widetilde{\varphi} \circ \widetilde{g}^{\prime}-\widetilde{g} \circ \widetilde{\varphi}\right\}(x, y) \in Z^{4}\left(\begin{array}{c}
\Omega \\
I
\end{array}\right)
\end{gathered}
$$

for any $(x, y) \in C^{2}$ in each case, if we choose $A, B, C, D$ and $\widetilde{\varphi}$ as follows: In the case of type (1),

$$
\begin{gathered}
A=\left(\begin{array}{ll}
a_{1} & 0 \\
0 & a_{2}
\end{array}\right) \quad B=\left(\begin{array}{ll}
b_{1} & 0 \\
0 & b_{2}
\end{array}\right) \quad C=\left(\begin{array}{ll}
c_{1} & 0 \\
0 & c_{2}
\end{array}\right) \quad D=\left(\begin{array}{ll}
d_{1} & 0 \\
0 & d_{2}
\end{array}\right) \\
\widetilde{\varphi}:(x, y) \mapsto(x, y)(C \Omega+D) .
\end{gathered}
$$

In the case of type (2),

$$
\begin{gathered}
A=\left(\begin{array}{cc}
a_{1} & c_{2} / 2 \\
0 & a_{2}
\end{array}\right) \quad B=\left(\begin{array}{cc}
b_{1} & \left(d_{2}-a_{1}\right) / 2 \\
0 & b_{2}
\end{array}\right) \quad C=\left(\begin{array}{cc}
c_{1} & 0 \\
0 & c_{2}
\end{array}\right) \quad D=\left(\begin{array}{cc}
d_{1} & -c_{1} / 2 \\
0 & d_{2}
\end{array}\right) \\
\tilde{\varphi}:(x, y) \mapsto(x, y)(C \Omega+D)+\left(0, c_{1} / 8\right) .
\end{gathered}
$$

In the case of types (3), (5) and (7),

$$
\begin{gathered}
A=\left(\begin{array}{ll}
a_{1} & 0 \\
0 & 1
\end{array}\right) \quad B=\left(\begin{array}{ll}
b_{1} & 0 \\
0 & 0
\end{array}\right) \quad C=\left(\begin{array}{ll}
c_{1} & 0 \\
0 & 0
\end{array}\right) \quad D=\left(\begin{array}{ll}
d_{1} & 0 \\
0 & 1
\end{array}\right) \\
\widetilde{\Phi}:(x, y) \mapsto(x, y)(C \Omega+D) .
\end{gathered}
$$

In the case of type (4),

$$
\begin{gathered}
A=\left(\begin{array}{cc}
a_{1} & -1 / 3 \\
0 & 1
\end{array}\right) \quad B=\left(\begin{array}{cc}
b_{1} & \left(a_{1}-1\right) / 3 \\
0 & 0
\end{array}\right) \quad C=\left(\begin{array}{cc}
c_{1} & c_{1} / 3 \\
0 & 0
\end{array}\right) \quad D=\left(\begin{array}{cc}
d_{1} & -c_{1} / 3 \\
0 & 1
\end{array}\right) \\
\widetilde{\Phi}:(x, y) \mapsto(x, y)(C \Omega+D)+\left(0, c_{1} / 9\right) .
\end{gathered}
$$

In the case of type (6),

$$
A=\left(\begin{array}{cc}
a_{1} & \left(1-a_{1}\right) / 2 \\
0 & 1
\end{array}\right) \quad B=\left(\begin{array}{cc}
b_{1} & \left(1-a_{1}\right) / 2 \\
0 & 0
\end{array}\right) \quad C=\left(\begin{array}{cc}
c_{1} & -c_{1} / 2 \\
0 & 0
\end{array}\right)
$$




$$
\begin{aligned}
D= & \left(\begin{array}{cc}
d_{1} & -c_{1} / 2 \\
0 & 1
\end{array}\right) \\
& \tilde{\varphi}:(x, y) \mapsto(x, y)(C \Omega+D)+\left(0, c_{1} \sigma_{4} / 8\right) .
\end{aligned}
$$

q.e.d.

Any hyperelliptic surface is an elliptic bundle in the following unique way.

$$
\begin{aligned}
& \varpi: S_{i}(\tau, \omega) \ni[x, y] \mapsto[2 x] \in E(2 \tau) \quad i=1,2 \\
& \varpi: S_{i}(\tau) \ni[x, y] \mapsto[N x] \in E(N \tau) \quad i=3,4,5,6,7,
\end{aligned}
$$

where the fibers of $\widetilde{\sigma}$ are $E(\omega), E\left(\sigma_{3}\right), E\left(\sigma_{4}\right)$ or $E\left(\sigma_{3}\right)$ in the case of type (1)(2), (3)(4), (5)(6) or (7), respectively. We easily see that $\widetilde{\sigma}$ has (1) four sections, (2) four 2-fold quasi-sections, (3) three sections, (4) three 3-fold quasi-sections, (5) two sections and a 2-fold quasi-section, (6) two 2-fold quasi-sections and a 4-fold quasi-section, (7) a section, a 2 -fold quasi-section and a 3 -fold quasi-section.

Now to kill automorphisms we consider hyperelliptic surfaces with specific base points. $o$ and $q$ are base points, while $p$ is a collection of zero, one, two or three base points depending on the types. We require $o, p$ and $q$ to satisfy the following conditions which we call (*) for simplicity:

In the case of type (1), $o$ is a point on one of the four sections of

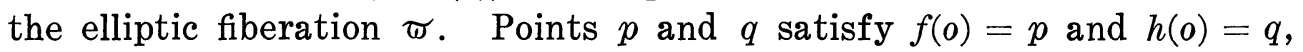
where $f$ and $h$ are elements of $\operatorname{Aut}(S)^{\circ}$ and $\operatorname{Aut}\left(\widetilde{\sigma}^{-1}(\widetilde{\sigma}(o))\right)^{\circ}$, respectively, of order 4 with $f^{2}(o)=o$.

In the case of type (2), $o$ is a point on one of the four 2-fold quasi-

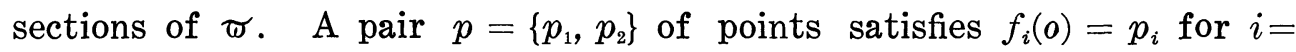
1,2 , where $f_{i}$ is an element of $\operatorname{Aut}(S)^{\circ}$ of order 4 such that $f_{i}(o)=o$ and that $f_{1} \neq f_{2}^{ \pm 1}$. A point $q$ satisfies $h(o)=q$ for an element $h$ of $\operatorname{Aut}\left(\widetilde{\varpi}^{-1}(\widetilde{\varpi}(o))\right)^{\circ}$ of order 4 .

In the case of type (3), $o$ is a point on one of the three sections of

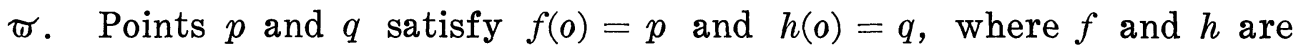
elements of $\operatorname{Aut}(S)^{\circ}$ and $\operatorname{Aut}\left(\widetilde{\sigma}^{-1}(\widetilde{\varpi}(o))\right)^{\circ}$ of order 6 and 2, respectively, with $f^{2}(o)=o$.

In the case of type (4), $o$ is a point on one of the three 3 -fold

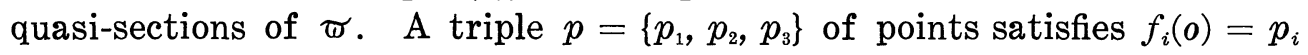
for $i=1,2,3$, where $f_{i}$ 's are mutually different elements of $\operatorname{Aut}(S)^{\circ}$ of order 9 with $f_{i}^{3}(o)=o$, and $f_{1} \circ f_{2} \circ f_{3}(o)=o$. A point $q$ satisfies $h(o)=q$ for an element $h$ of $\operatorname{Aut}\left(\widetilde{\sigma}^{-1}(\widetilde{\sigma}(o))\right)^{\circ}$ of order 2.

In the case of type (5), $o$ is a point on one of the two sections of 
$\varpi$. A point $q$ satisfies $h(o)=q$, where $h$ is an element of $\operatorname{Aut}\left(\widetilde{\sigma}^{-1}(\widetilde{\sigma}(o))\right)^{\circ}$ of order 4 such that $h^{2}(0)$ is on a section of $\varpi$.

In the case of type (6), $o$ is a point on one of the two 2-fold quasi-

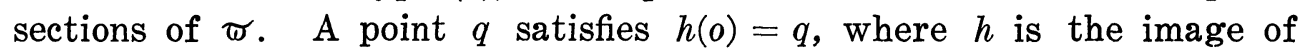

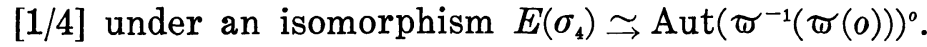

In the case of type (7), $o$ is a point on the section of $\varpi$. A point

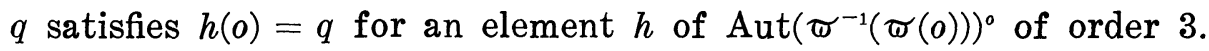

We denote the hyperelliptic surface $S$ with these base points by $S(o, p, q)$ or $S(o, q)$.

Definition. By a family $(\pi: \mathscr{S} \rightarrow T ; \mathfrak{p}, \mathfrak{p}, \mathfrak{q})$ of hyperelliptic surfaces of type (1) with base points over an analytic space $T$, we mean a flat $\operatorname{map} \pi: \mathscr{S} \rightarrow T$ of analytic spaces together with sections $\mathfrak{o}, \mathfrak{p}$ and $\mathfrak{q}$ such that the fibers $\left(\pi^{-1}(t) ; \mathfrak{D}(t), \mathfrak{p}(t), \mathfrak{q}(t)\right)$ are hyperelliptic surfaces of type $(1)$ with base points satisfying the property $(*)$ for $o=\mathfrak{o}(t), \quad p=\mathfrak{p}(t)$ and $q=\mathfrak{q}(t)$.

We can define a family of hyperelliptic surfaces of type (2), (3), (4), (5), (6) or (7) with base points in the same way as above.

On $S_{i}(\tau, \omega)$ and $S_{i}(\tau)$, we can choose base points $o^{*}, p^{*}$ and $q^{*}$ satisfying these requirements $(*)$ as follows:

In the case of type (1),

$$
o^{*}=[0,0], \quad p^{*}=[1 / 4,0], \quad q^{*}=[0,1 / 4] .
$$

In the case of type (2),

$$
o^{*}=[0,0], \quad p^{*}=\{[1 / 4,0],[1 / 4+\tau, 0]\}, \quad q^{*}=[0,1 / 4] .
$$

In the case of type (3),

$$
o^{*}=[0,0], \quad p^{*}=[1 / 6,0], \quad q^{*}=[0,1 / 2] .
$$

In the case of type (4),

$$
\begin{aligned}
& o^{*}=[0,0], \quad p^{*}=\{[1 / 9,0],[1 / 9+\tau, 0],[1 / 9+2 \tau, 0]\}, \\
& q^{*}=[0,1 / 2] .
\end{aligned}
$$

In the case of type (5),

$$
o^{*}=[0,0], \quad q^{*}=\left[0,\left(1+\sigma_{4}\right) / 4\right] .
$$

In the case of type (6),

$$
o^{*}=[0,0], \quad q^{*}=[0,1 / 4] .
$$

In the case of type (7),

$$
o^{*}=[0,0], \quad q^{*}=[0,1 / 3] .
$$


Then we can verify the following:

REMARK 4. Any automorphism of $S_{i}(\tau, \omega)$ or $S_{i}(\tau)$ which fixes $o^{*}, p^{*}$ and $q^{*}$, is the identity.

We get the following proposition by considerations similar to those in Proposition 1.

Proposition 3. The set of isomorphism classes of hyperelliptic surfaces of type $(i)$ with base points is in one to one correspondence with $M_{i}$ defined as follows:

$$
\begin{aligned}
& M_{1}=M_{2}=\left(\mathfrak{S} / \Gamma_{o}(4)\right)^{2}, \quad M_{3}=\mathfrak{S} / \Gamma_{o}(6), \quad M_{4}=\mathscr{S} / \Gamma_{o}(9), \\
& M_{5}=\mathscr{S} / \Gamma_{o}(4), \quad M_{6}=\mathfrak{S}_{\mathcal{C}} / \Gamma_{o}(8), \quad M_{7}=\mathfrak{S}_{\mathcal{C}} / \Gamma_{o}(6) .
\end{aligned}
$$

REMARK 5. There exists a family over $M_{i}$ of hyperelliptic surfaces of type $(i)$ with base points effectively parametrized and complete. Indeed, let

$$
\left(\begin{array}{ll}
a & b \\
c & d
\end{array}\right), \quad\left(\begin{array}{ll}
e & f \\
g & h
\end{array}\right) \in \bar{\Gamma}_{o}(N)
$$

which are mapped to $\gamma, \delta \in \Gamma_{o}(N)$, respectively, and let

$$
\begin{aligned}
(\gamma, \delta):[x, y, \tau, \omega] & \mapsto\left[\frac{x}{c \tau+d}, \frac{y}{g \omega+h}, \gamma(\tau), \delta(\omega)\right] \text { for } i=1,2, \\
\gamma:[x, y, \tau] & \mapsto\left[\frac{x}{c \tau+d}, y, \gamma(\tau)\right] \text { for } i=3,4,5,6,7 .
\end{aligned}
$$

Then $\left(\Gamma_{o}(4)\right)^{2},\left(\Gamma_{o}(4)\right)^{2}, \Gamma_{o}(6), \Gamma_{o}(9), \Gamma_{o}(4), \Gamma_{o}(8)$ and $\Gamma_{o}(6)$ act on $\tilde{\mathscr{S}}_{i}$ for $i=1,2,3,4,5,6$ and 7 , respectively without fixed point, and the actions commute with $\tilde{\pi}_{i}$. Let

$$
\begin{array}{ll}
\mathscr{S}_{i}=\tilde{\mathscr{S}}_{i} \mid\left(\Gamma_{0}(4)\right)^{2} & i=1,2 \quad \tilde{\mathscr{S}}_{3}=\tilde{\mathscr{S}}_{3} \mid \Gamma_{0}(6) \\
\mathscr{S}_{4}=\tilde{\mathscr{S}}_{4} \mid \Gamma_{0}(9) & \mathscr{S}_{5}=\tilde{\mathscr{S}}_{5} \mid \Gamma^{o}(4) \\
\mathscr{S}_{6}=\tilde{\mathscr{S}}_{6} \mid \Gamma_{0}(8) & \mathscr{S}_{7}=\tilde{\mathscr{S}}_{7} \mid \Gamma_{0}(6),
\end{array}
$$

and let

$$
\pi_{i}: \mathscr{S}_{i} \rightarrow M_{i}
$$

be the holomorphic map induced by $\tilde{\pi}_{i}$. Then $\pi_{i}$ is smooth and $\pi_{i}^{-1}([\tau, \omega]) \cong S_{i}(\tau, \omega) \quad i=1,2$ or $\pi_{i}^{-1}([\tau]) \cong S_{i}(\tau) \quad i=3,4,5,6,7$, where $[\tau, \omega]$ or $[\tau]$ denotes the points of $M_{i}$ which is the image of $(\tau, \omega)$ in $\mathfrak{S}^{2}$ or $\tau$ in $\mathfrak{S}$, respectively. Moreover, let $\mathfrak{D}^{*}, \mathfrak{p}^{*}$ and $\mathfrak{q}^{*}$ be the maps which send each point $t$ of $M_{i}$ to $o^{*}, p^{*}$ and $q^{*}$ of the fiber $\pi_{i}^{-1}(t)$, respectively. Then $\mathfrak{o}^{*}, \mathfrak{p}^{*}$ and $\mathfrak{q}^{*}$ are sections or sections and a quasi-section of $\pi_{i}$ de- 
pending on $i$.

THEOREM 2. $\pi_{i}: \mathscr{S}_{i} \rightarrow M_{i}$ is the universal family of hyperelliptic surfaces of type (i) with base points, i.e., $M_{i}$ is the fine moduli space in the sense of Mumford [8] of hyperelliptic surfaces of type (i) with base points.

Proof. We prove the theorem only in the case of type (1). The proof for the other types are similar.

By Proposition 3, we have a unique map $f: T \rightarrow M_{1}$ for any family over an analytic space $T$ of hyperelliptic surfaces of type (1) with base points. We will see shortly that this map $f$ is holomorphic. Then we have a morphism $\Phi_{1}: \mathfrak{M}_{1} \rightarrow h_{M_{1}}$ of contravariant functors, where $\mathfrak{M}_{1}$ and $h_{M_{1}}$ denote the set of families of hyperelliptic surfaces over $T$ of type (1) with base points, modulo isomorphism, and the set of holomorphic maps from $T$ to $M_{1}$, respectively. By Remark 5, $\Phi_{1}(T): \mathfrak{M}_{1}(T) \rightarrow h_{M_{1}}(T)$ is surjective for any analytic space $T$. Thus it is enough to show that for any family $(\pi: \mathscr{S} \rightarrow T ; \mathfrak{v}, \mathfrak{p}, \mathfrak{q})$ of hyperelliptic surfaces of type (1) with base points, there is an isomorphism from $\varphi$ to $\mathscr{S}_{1} \times_{M_{1}} T$ over $T$, which maps $\mathfrak{p}(t), \mathfrak{p}(t)$ and $\mathfrak{q}(t)$ to $\left(\mathfrak{o}^{*} \circ f(t), t\right),\left(\mathfrak{p}^{*} \circ f(t), t\right)$ and $\left(\mathfrak{q}^{*} \circ f(t), t\right)$, respectively, for each point $t$ of $T$. For any point $t_{0}$ of $T, \pi_{1}^{-1}\left(f\left(t_{0}\right)\right) \cong$ $\pi^{-1}\left(t_{o}\right)$. Hence, by Remark 5 , there is a holomorphic map $f^{\prime}$ from a connected neighborhood $U$ of $t_{o}$ to $M_{1}$ with $f^{\prime}\left(t_{o}\right)=f\left(t_{o}\right)$ and an isomorphism

$$
F^{\prime}: \mathscr{S}_{1 U} \underset{\sim}{\longrightarrow} \mathscr{S}_{1} \times_{M_{1}} U
$$

over $U$ such that $F^{\prime} \circ \mathfrak{p}\left(t_{o}\right)=\left(\mathfrak{o}^{*} \circ f^{\prime}\left(t_{o}\right), t_{o}\right), F^{\prime} \circ \mathfrak{p}\left(t_{o}\right)=\left(\mathfrak{p}^{*} \circ f^{\prime}\left(t_{o}\right), t_{o}\right)$ and $F^{\prime} \circ \mathfrak{q}\left(t_{o}\right)=\left(\mathfrak{q}^{*} \circ f^{\prime}\left(t_{o}\right), t_{o}\right)$. By Remarks 1 and $5, \mathscr{S}_{1} \times_{M_{1}} U$ is expressed as a quotient manifold of $C^{2} \times U$. Hence there exists an automorphism of $\mathscr{S}_{1} \times_{M_{1}} U$ which is induced by an automorphism of $C^{2} \times U$ of the form $(x, y, t) \mapsto(x+a(t), y, t)$ and which maps $F^{\prime} \circ \mathfrak{o}(t)$ to ( $\left.{ }^{*} \circ f^{\prime}(t), t\right)$, where $a$ is a holomorphic function on $U$ vanishing at $t_{o}$. Composing this automorphism with $F^{\prime}$, we get an isomorphism

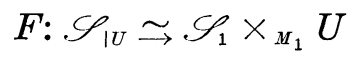

over $U$ which maps $\mathfrak{D}(t)$ to $\left(\mathfrak{o}^{*} f^{\prime}(t), t\right)$. For any $\tau, \omega \in \mathfrak{F}$, there exist only four points $p_{1}^{*}=p^{*}, p_{2}^{*}=[3 / 4,0], p_{3}^{*}=[(1+2 \tau) / 4,0]$ and $p_{4}^{*}=$ $\left[(3+2 \tau / 4,0]\right.$ on $S_{1}(\tau, \omega)$ such that $\left(o^{*}, p_{k}^{*}, ?\right)$ satisfy the property $(*)$. Therefore $F \circ \mathfrak{p}(t)$ agrees with one of these points $p_{k}^{*}$ on $\pi^{-1}(f(t)) \cong S_{1}(\tau, \omega)$, where $f^{\prime}(t)=[\tau, \omega]$. But these points $p_{k}^{*}$ for $k=1,2,3,4$ on each fiber $\pi^{-1}\left(f^{\prime}(t)\right) \cong S_{1}(\tau, \omega)$ form the sections

$$
\mathfrak{p}_{k}^{*}: U \rightarrow \mathscr{S}_{1} \times_{M_{1}} U \text { for } \quad k=1,2,3,4,
$$


respectively. Hence $F \circ \mathfrak{p}$ equals one of these sections $\mathfrak{p}_{k}^{*}$, since $U$ is connected. Thus $F \circ \mathfrak{p}=\left(\mathfrak{p}^{*} \circ f^{\prime}, \mathrm{id}\right)$ on $U$ for $F \circ \mathfrak{p}\left(t_{o}\right)=\left(\mathfrak{p}_{1}^{*} \circ f^{\prime}\left(t_{o}\right), t_{o}\right)$ and for $\mathfrak{p}^{*}=\mathfrak{p}_{1}^{*}$. By the same consideration, we get $F \circ \mathfrak{q}=\left(\mathfrak{q}^{*} \circ f^{\prime}\right.$, id $)$ on $U$. Therefore, $f=f^{\prime}$ and $F \circ \mathfrak{D}=\left(\mathfrak{o}^{*} \circ f, \mathrm{id}\right), F \circ \mathfrak{p}=\left(\mathfrak{p}^{*} \circ f, \mathrm{id}\right), F \circ \mathfrak{q}=\left(\mathfrak{q}^{*} \circ f\right.$, id) on $U$. Since $F$ is uniquely determined by $f$, we can, by Remark 5 , patch up these $F$ 's defined on neighborhoods $U$ 's which cover $T$. Thus we get a unique isomorphism from $\mathscr{S}$ to $\mathscr{S}_{1} \times_{M_{1}} T$ over $T$. q.e.d.

2. Since $\mathfrak{S} / \Gamma_{o}(N)$ is a complex manifold of dimension 1 , it has a unique non-singular compactification $\overline{\left(\mathcal{S}_{\mathrm{c}} / \Gamma_{o}(N)\right)}$, and when $N=4,6,8$ or 9 , it is biholomorphic to the projective line $\boldsymbol{P}^{1}$. We construct it explicitely, for later convenience. (See [7] I.)

There are $t \quad \Gamma_{o}(N)$-equivalence classes of the cusps for $\Gamma_{o}(N)$ acting on $\mathfrak{F}$, where $t$ is the same as in Proposition 1 . We choose representative points $p_{1}, p_{2}, \cdots, p_{t}$ from each of them. Let

$$
\begin{gathered}
\mathscr{S}_{p_{i}}=\left\{\omega \in \mathfrak{S}_{\mathcal{C}} \mid \operatorname{Im}(\delta(\omega))>C\right\}, \\
\Gamma_{p_{i}}=\delta^{-1} \Gamma_{\infty}(n) \delta, \\
\Gamma_{\infty}(n)=\left\{\left(\begin{array}{ll}
1 & b \\
0 & 1
\end{array}\right) \mid b \equiv 0(\bmod n)\right\} /\{ \pm I\},
\end{gathered}
$$

where $\delta \in \Gamma$ with $\delta\left(p_{i}\right)=\infty$, and $n$ is the smallest positive integer such that $\delta^{-1} \Gamma_{\infty}(n) \delta$ is the subgroup of $\Gamma_{o}(N)$. Then $\mathfrak{S}_{p_{i}} / \Gamma_{p_{i}}$ is isomorphic to a punctured disk $\Delta_{p_{i}}^{*}$, and we can regard $\Delta_{p_{i}}^{*}$ as an open set of $\mathfrak{S}_{2} / \Gamma_{o}(N)$ for sufficiently large $C$, since $\mathfrak{S}_{p_{i}}$ is a neighborhood of $p_{i}$, and $\Gamma_{p_{i}}$ is the stabilizer of $p_{i}$.

Let

$$
\overline{\left(\mathfrak{S} / \Gamma_{o}(N)\right)}=\mathscr{S} / \Gamma_{o}(N) \cup \Delta_{p_{1}} \cup \Delta_{p_{2}} \cup \cdots \cup \Delta_{p_{t}},
$$

by the natural identifications, where $\Delta_{p_{i}}$ is the disk with the same radius as $\Delta_{p_{i}}^{*}$. Then $\overline{\left(\mathfrak{S} / \Gamma_{o}(N)\right)}$ is the non-singular compactification of $\mathfrak{S} / \Gamma_{o}(N)$. Let

$$
\alpha_{p_{i}}: \mathfrak{S}_{p_{i}} \rightarrow \Delta_{p_{i}}^{*}
$$

be the projection. Then $\alpha_{p_{i}}(\omega)=\exp (2 \pi i \delta(\omega) / n)$. For $N=4,6,8,9$ we have the following:

$N=4$. The cusps of $\Gamma_{0}(4)$ are represented by $\infty, 0$ and $1 / 2$, and $\alpha_{\infty}(\omega)=\boldsymbol{e}(\omega), \alpha_{0}(\omega)=\boldsymbol{e}(-1 / 4 \omega), \alpha_{1 / 2}(\omega)=\boldsymbol{e}(\alpha /(1-2 \omega)), \quad$ where $\quad \boldsymbol{e}(\omega)=$ $\exp (2 \pi i \omega)$.

$N=6$. The cusps of $\Gamma_{o}(6)$ are represented by $\infty, 0,1 / 2$ and $1 / 3$, and $\quad \alpha_{\infty}(\omega)=\boldsymbol{e}(\omega), \alpha_{0}(\omega)=\boldsymbol{e}(-1 / 6 \omega), \alpha_{1 / 2}(\omega)=\boldsymbol{e}(\omega / 3(1-2 \omega)), \quad \alpha_{1 / 3}(\omega)=$ $\boldsymbol{e}(\omega / 2(1-3 \omega))$. 
$N=8$. The cusps of $\Gamma_{o}(8)$ are represented by $\infty, 0,1 / 2$ and $1 / 4$, and $\alpha_{\infty}(\omega)=\boldsymbol{e}(\omega), \alpha_{0}(\omega)=\boldsymbol{e}(-1 / 8 \omega), \alpha_{1 / 2}(\omega)=\boldsymbol{e}(\omega /(1-2 \omega)), \alpha_{1 / 4}(\omega)=\boldsymbol{e}(\omega /(1-4 \omega))$.

$N=9$. The cusps of $\Gamma_{o}(9)$ are represented by $\infty, 0,1 / 3$ and $-1 / 3$, and $\quad \alpha_{\infty}(\omega)=\boldsymbol{e}(\omega), \quad \alpha_{0}(\omega)=\boldsymbol{e}(-1 / 9 \omega), \quad \alpha_{1 / 3}(\omega /(1-3 \omega))=\boldsymbol{e}(\omega /(1-3 \omega))$, $\alpha_{-1 / 3}(\omega)=e(\omega /(1+3 \omega))$.

Definition. $\quad \bar{M}_{1}=\bar{M}_{2}=\overline{\left(\mathfrak{S} / \Gamma_{o}(4)\right)^{2}} \quad \bar{M}_{3}=\overline{\left(\mathfrak{S} / \Gamma_{o}(6)\right)} \quad \bar{M}_{4}=\overline{\left(\mathfrak{S} / \Gamma_{o}(9)\right)} \quad \bar{M}_{5}=$ $\overline{\left(\mathfrak{S} / \Gamma_{o}(4)\right)} \quad \bar{M}_{6}=\overline{\left(\mathfrak{S} / \Gamma_{o}(8)\right)} \bar{M}_{7}=\overline{\left(\mathfrak{S} / \Gamma_{o}(6)\right)}$.

When $i=3$ through $7, \bar{M}_{i}$ is the unique compactification of $M_{i}$, but $\bar{M}_{1}$ or $\bar{M}_{2}$ is only one of many other possible compactifications of $M_{1}$ or $M_{2}$, respectively.

Our final goal is to show that a possibly degenerate hyperelliptic surface with base points "naturally" corresponds to each point of $\bar{M}_{i}$ in the following way: For any point $t_{o}$ of $\bar{M}_{i}$, there exists a neighborhood $U$ of $t_{o}$, a finite covering $\varphi: V \rightarrow U$ with $\varphi^{-1}\left(t_{o}\right)$ consisting of a point $s_{o}$, and a family $\pi: \mathscr{S} \rightarrow V$ with sections $\mathfrak{D}, \mathfrak{q}$ and a section or a quasisection $\mathfrak{p}$ such that

$$
\mathscr{P}_{\mid \varphi^{-1}\left(U \cap M_{i}\right)} \underset{\rightarrow}{\rightarrow} \mathscr{P}_{i} \times_{M_{i}} \varphi^{-1}\left(U \cap M_{i}\right)
$$

over $\varphi^{-1}\left(U \cap M_{i}\right)$, where $\pi_{i}: \mathscr{S}_{i} \rightarrow M_{i}$ is the family in Remark 5 . In this case, we say that $\pi^{-1}\left(s_{o}\right)\left(\mathfrak{D}\left(s_{o}\right), \mathfrak{p}\left(s_{o}\right), \mathfrak{q}\left(s_{o}\right)\right)$ corresponds to $t_{o}=\varphi\left(s_{o}\right)$.

It is enough for our purpose to construct families with base points over finite covering spaces of $\left(\mathfrak{E} / \Gamma_{o}(4)\right) \times \Delta_{d}, \Delta_{d} \times\left(\mathfrak{E} / \Gamma_{o}(4)\right)$ and $\Delta_{d} \times \Delta_{d^{\prime}}$ or $\Delta_{d}$ 's, which together with $M_{i}$ cover $\bar{M}_{i}$, when $i=1,2$ or $i=3$ through 7 , respectively. We construct these families in the next section as follows: First, we construct degenerating families $\mathscr{A}$ of abelian surfaces and automorphisms $\mathfrak{g}$ of $\mathscr{A}$. Secondly, we construct, if possible, a non-singular model $\mathscr{S}$, flat over the base space, of $\mathscr{A} / \mathfrak{g}^{z}$. Finally, we add base sections to these families.

For these constructions, we need the following. We use the theory of torus embeddings in the same notations as in Oda [5]. Especially we denote by $\varphi_{*}$ the morphism of torus embeddings which corresponds to a morphism $\varphi$ of r.p.p. decompositions.

(I) Families of abelian surfaces

(1) Let $N=Z^{4}$ with $Z$-basis $\left\{n_{1}, n_{2}, n_{3}, n_{4}\right\}$, and let $N^{\prime}=Z^{2}$ with $Z$-basis $\left\{n_{1}^{\prime}, n_{2}^{\prime}\right\}$. Let $X=T_{N}$ emb $(\Sigma)$, where $\Sigma=$ all the faces of $\sigma_{i, j} \mid i$, $j \in \boldsymbol{Z}\}$ with

$\sigma_{i, j}=\boldsymbol{R}_{0}\left(n_{3}+i n_{1}\right)+\boldsymbol{R}_{0}\left(n_{3}+(i+1) n_{1}\right)+\boldsymbol{R}_{0}\left(n_{4}+j n_{2}\right)+\boldsymbol{R}_{0}\left(n_{4}+(j+1) n_{2}\right)$.

Then $X$ is non-singular. On the other hand, let $Y=T_{N}$, emb( $\left.\Lambda\right)$, where 
$A=\left\{\right.$ the faces of $\left.\boldsymbol{R}_{0} n_{1}^{\prime}+\boldsymbol{R}_{0} n_{2}^{\prime}\right\}$. Then $Y \cong \boldsymbol{C}^{2}$. Let $\phi$ be the morphism of r.p.p. decompositions from $(N, \Sigma)$ to $\left(N^{\prime}, \Lambda\right)$ defined by

$$
\varphi: \sum_{i=1}^{4} a_{i} n_{i} \mapsto a_{3} n_{1}^{\prime}+a_{4} n_{2}^{\prime} .
$$

Thus we have an equivariant morphism $\varphi_{*}: X \rightarrow Y \cong C^{2}$. Let $\lambda, \kappa, \nu$ and $\mu$ be the automorphisms of $(N, \Sigma)$ defined by

$$
\begin{aligned}
& \lambda: \sum_{i=1}^{4} a_{i} n_{i} \mapsto\left(a_{1}+a_{3}\right) n_{1}+\sum_{i=2}^{4} a_{i} n_{i} \\
& \kappa: \sum_{i=1}^{4} a_{i} n_{i} \mapsto\left(a_{2}+a_{4}\right) n_{2}+\sum_{i=1,3,4} a_{i} n_{i} \\
& \nu: \sum_{i=1}^{4} a_{i} n_{i} \mapsto-a_{1} n_{1}+\sum_{i=2}^{4} a_{i} n_{i} \\
& \mu: \sum_{i=1}^{4} a_{i} n_{i} \mapsto-a_{2} n_{2}+\sum_{i=1,3,4} a_{i} n_{i} .
\end{aligned}
$$

Then $\varphi_{*} \circ \lambda_{*}=\varphi_{*}, \varphi_{*} \circ \kappa_{*}=\varphi_{*}, \varphi_{*} \circ \nu_{*}=\varphi_{*}$ and $\varphi_{*} \circ \mu_{*}=\varphi_{*}$. Let $\theta$ and $\rho$ be the extensions to $T_{N} \mathrm{emb}(\Sigma)$ of the actions $(v, w, s, t) \mapsto(-v, w, s, t)$ and $(v, w, s, t) \mapsto(v,-w, s, t)$ of $T_{N}$, respectively. Let

$$
\begin{array}{ll}
\mathscr{A}_{1}=X_{\mid D} / \lambda_{*}^{Z} \times \kappa_{*}^{2 Z} & \mathscr{A}_{2}=X_{\mid D} / \lambda_{*}^{4 Z} \times \kappa_{*}^{2 Z} \\
\mathscr{A}_{3}=X_{\mid D} / \lambda_{*}^{Z} \times \kappa_{*}^{4 Z} \quad \mathscr{A}_{4}=X_{\mid D} / \lambda_{*}^{4 Z} \times \kappa_{*}^{4 Z} \\
\mathscr{A}_{5}=X_{\mid D} /\left(\rho \circ \lambda_{*}\right)^{Z} \times \kappa_{*}^{2 Z} \quad \mathscr{A}_{6}=X_{\mid D} /(\theta \circ \rho)^{Z} \times \lambda_{*}^{2 Z} \times \kappa_{*}^{2 Z} \\
\mathscr{A}_{7}=X_{\mid D} /\left(\lambda_{*} \circ \kappa_{*}^{2}\right)^{Z} \times \kappa_{*}^{4 Z} & \mathscr{A}_{8}=X_{\mid D} / \lambda_{*}^{2 Z} \times\left(\theta \circ \kappa_{*}^{2}\right)^{z},
\end{array}
$$

where $D$ is the unit polydisk in $T_{N^{\prime}} \operatorname{emb}(\Lambda)$ and $X_{\mid D}=\varphi_{*}^{-1}(D)$. When $i \neq 6, \mathscr{A}_{i}$ is non-singular, but $\mathscr{A}_{6}$ has four isolated singular points some neighborhoods of which are isomorphic to $\operatorname{Spec}\left\{C\left[x^{2}, y^{2}, z^{2}, w^{2}, x y, x z, x w\right.\right.$, $y z, y w, z w]\}$. If an automorphism $\varepsilon$ of $X$ induces an automorphism of $\mathscr{A}_{i}$ by natural projection $X \rightarrow \mathscr{A}_{i}$, we denote the induced automorphism by $[\varepsilon]$. Let $\widetilde{\sigma}_{i}: \mathscr{A}_{i} \rightarrow D$ be the holomorphic map induced by $\varphi_{*}$. Then for non-zero $s$ and $t, \widetilde{\sigma}_{i}^{-1}(s, t)$ is an abelian surface, while $\widetilde{\sigma}_{i}^{-1}(s, 0)$ and $\widetilde{\sigma}_{i}^{-1}(0, t)$ consist of components each of which is isomorphic to a product $E \times \boldsymbol{P}^{1}$ of an elliptic curve and a line. These components cross along elliptic curves. When $i \neq 6, \widetilde{\sigma}_{i}^{-1}(0,0)$ consists of components each of which is isomorphic to $\boldsymbol{P}^{1} \times \boldsymbol{P}^{1}$, and these components intersect along fibers and sections. $\widetilde{\sigma}_{6}^{-1}(0,0)$ consists of four components each of which is isomorphic to

$$
V=\boldsymbol{P}^{1} \times \boldsymbol{P}^{1} / h^{z} \quad \text { with } \quad h:(\eta, \xi) \mapsto(-\eta,-\xi) .
$$

(2) Let $\phi_{n}: \mathscr{C}_{n} \rightarrow \Delta=\{s \in C|| s \mid<1\}$ be the family of elliptic curves, whose general fiber $\phi_{n}^{-1}(s)$ has the periods 1 and $n(\log s) / 2 \pi i$, and $\phi_{n}^{-1}(0)$ 

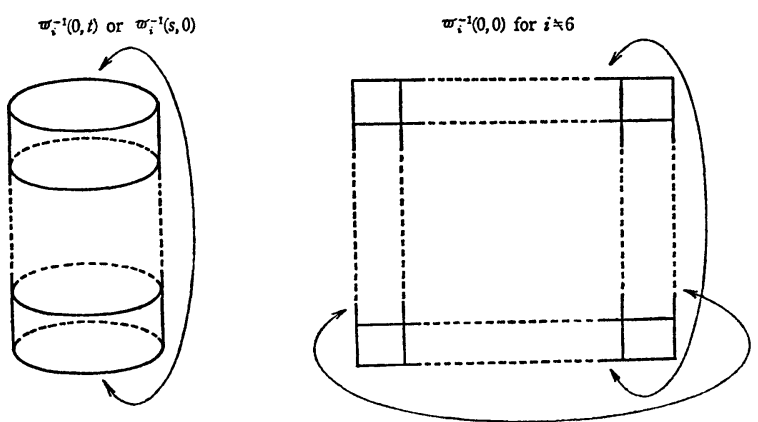

Figure 2-1

is a cycle of $n$ rational curves crossing normally. Let $\alpha_{k}, \beta$ and $\gamma$ be the automorphisms of $\mathscr{E}_{n}$ defined by

$$
\begin{aligned}
\alpha_{k}:(w, s) & \mapsto\left(\sigma_{k} w, s\right) \quad \text { with } \quad \sigma_{k}=\exp (2 \pi i / k), \\
\beta:(w, s) & \mapsto(s w, s), \quad \gamma:(w, s) \mapsto\left(w^{-1}, s\right),
\end{aligned}
$$

respectively. Let

$$
\begin{gathered}
\widetilde{\mathscr{S}}_{j}=\boldsymbol{C} \times \mathscr{S} /\left\{h_{a}^{j} \mid a \in \boldsymbol{Z}^{2}\right\} \text { with } \\
h_{\left(a_{1}, a_{2}\right)}^{1}:(x, \tau) \mapsto\left(x+a_{1} \tau+a_{2}, \tau\right) \\
h_{\left(a_{1}, a_{2}\right)}^{2}:(x, \tau) \mapsto\left(x+2 a_{1} \tau+a_{2}, \tau\right) .
\end{gathered}
$$

For any element $\delta \in \bar{\Gamma}_{o}(4)$, we define the automorphism of $\widetilde{\mathscr{B}}_{i}$ by

$$
[x, \tau] \mapsto[x /(c \tau+d), \tau], \quad \text { with } \delta=\left(\begin{array}{ll}
a & b \\
c & d
\end{array}\right) .
$$

Then $\bar{\Gamma}_{o}(4)$ acts on $\tilde{\mathscr{B}}_{i}$. Let $\mathscr{\mathscr { B }}_{i}=\tilde{\mathscr{B}}_{i} / \bar{\Gamma}_{o}(4)$ and let $\psi_{i}: \mathscr{B}_{i} \rightarrow\left(\mathscr{S} / \Gamma_{o}(4)\right)$ be the holomorphic map induced by the projection $C \times \mathfrak{S} \rightarrow \mathfrak{S}$. Clearly $\phi_{k} \times \psi_{i}: \mathscr{E}_{k} \times \mathscr{B}_{i} \rightarrow \Delta \times\left(\mathscr{S}_{\mathfrak{C}} / \Gamma_{o}(4)\right)$ is a degenerating families of abelian surfaces.

(II) The resolution of quotient singularities.

We consider the following singularity. For a positive integer $b$, let

$$
N_{b}=C^{3} / h^{z} \quad \text { with } \quad h:(x, y, z) \mapsto\left(\sigma_{b} x, \sigma_{b}^{-1} y, \sigma_{b}^{-1} z\right),
$$

where $\sigma_{b}=\exp (2 \pi i / b)$. Ueno [12] constructed the canonical resolution of this singularity. Using torus embeddings, we reconstruct this resolution endowed with a fiberation different from that considered in [12].

Let $N=Z^{3}$ with $Z$-basis $\left\{n_{1}, n_{2}, n_{3}\right\}$, and let $n_{1}^{\prime}=b n_{1}+n_{2}+n_{3}$, $n_{2}^{\prime}=n_{2}, n_{3}^{\prime}=n_{3}$. Let

$$
\nabla=\{\text { the faces of } \sigma\} \text {, with } \sigma=\boldsymbol{R}_{0} n_{1}^{\prime}+\boldsymbol{R}_{0} n_{2}^{\prime}+\boldsymbol{R}_{0} n_{3}^{\prime},
$$




$$
\begin{aligned}
& \tilde{\nabla}=\left\{\text { the faces of } \sigma_{0}, \sigma_{k} \text { and } \tau_{k} \mid k=1, \cdots, b-1\right\} \text { with } \\
& \sigma_{0}=\boldsymbol{R}_{0}\left(n_{1}+n_{2}+n_{3}\right)+\boldsymbol{R}_{0} n_{2}+\boldsymbol{R}_{0} n_{3} \\
& \boldsymbol{\sigma}_{k}=\boldsymbol{R}_{0}\left(k n_{1}+n_{2}+n_{3}\right)+\boldsymbol{R}_{0}\left((k+1) n_{1}+n_{2}+n_{3}\right)+\boldsymbol{R}_{0} n_{2} \\
& \tau_{k}=\boldsymbol{R}_{0}\left(k n_{1}+n_{2}+n_{3}\right)+\boldsymbol{R}_{0}\left((k+1) n_{1}+n_{2}+n_{3}\right)+\boldsymbol{R}_{0} n_{3} .
\end{aligned}
$$

Then $\tilde{\nabla}$ is a subdivision of $\nabla$, and $T_{N} \operatorname{emb}(\tilde{\nabla})$ is non-singular. $T_{N} \mathrm{emb}(\nabla)$ and $T_{N} \mathrm{emb}(\tilde{\nabla})$ are isomorphic to $N_{b}$ and $M$ in [12], respectively. $\iota_{*}$ agrees with $T^{-1}$ in [12], where $\iota_{*}$ is the holomorphic map induced by the identity map $\iota$ of $N$. See Figure 2-2.
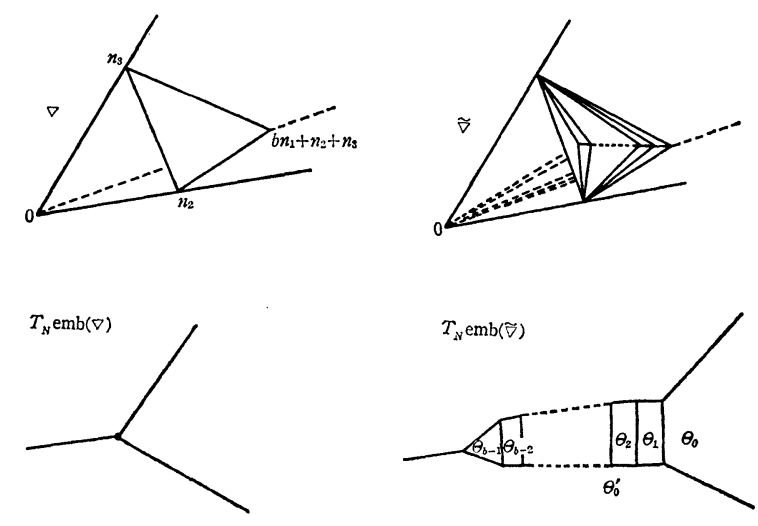

Figure 2-2

Next let $L=\boldsymbol{Z}$ with $\boldsymbol{Z}$-basis $l$, and let $\square=\left\{0, \boldsymbol{R}_{0} l\right\}$. Let $\pi$ be the morphism of r.p.p. decompositions defined by

$$
\pi:(N, \tilde{\nabla}) \ni a_{1} n_{1}+a_{2} n_{2}+a_{3} n_{3} \mapsto a_{2} l \in(L, \square) .
$$

Then we obtain

$$
\pi_{*}^{-1}\left(\operatorname{orb}\left(\boldsymbol{R}_{0} l\right)\right)=\sum_{k=0}^{b-1} \Theta_{k}+\Theta_{0}^{\prime},
$$

where $\left.\Theta_{k}=\overline{\operatorname{orb}\left(\boldsymbol{R}_{0}\left((b-k) n_{1}+n_{2}+n_{3}\right)\right.}\right), \Theta_{0}^{\prime}=\overline{\operatorname{orb}\left(\boldsymbol{R}_{0} n_{2}\right)}$. We easily see that $\Theta_{k}$ and $\Theta_{0}^{\prime}$ are isomorphic to those in [12], and intersect in the same way as in Lemma 4.6 [12]. In particular, we note that $\Theta_{b-1} \cong \boldsymbol{P}^{2}$ and $\Theta_{k} \cong \Sigma_{b-k}$ for $1 \leqq k \leqq b-2$, where $\Sigma_{d}$ is the $\boldsymbol{P}^{1}$-bundle over $\boldsymbol{P}^{1}$ of degree $d$.

3. We use the local degenerating families $\widetilde{\sigma}_{i}: \mathscr{A}_{i} \rightarrow D, i=1$ through $8, \phi_{k} \times \psi_{j}: \mathscr{E}_{k} \times \mathscr{B}_{j} \rightarrow \Delta \times\left(\mathscr{S}_{\mathcal{C}} / \Gamma_{o}(4)\right), k=1$ through $4, j=1,2$, and $\phi_{k}: \mathscr{E}_{k} \times E \rightarrow$ $\Delta, k=1$ through 6 , defined in the previous section. Most of the components of "degenerate hyperelliptic surfaces" described in this section are elliptic surfaces. In describing their singular fibers, we use the notation of Kodaira [4]. In the following, we denote by $\widetilde{\Delta} \rightarrow \Delta$, sending 
$s$ to $s^{2}$, the double covering of a disk $\Delta$.

(1-i) Over $\Delta_{\infty} \times\left(S_{\mathfrak{C}} / \Gamma_{o}(4)\right)$.

Let $\mathscr{A}=\mathscr{E}_{1} \times \mathscr{B}_{1}$ and

$$
\mathrm{g}: \mathscr{A} \ni(p,[y, \omega]) \mapsto\left(\alpha_{2} p,[-y, \omega]\right) \in \mathscr{L} .
$$

Then $g$ has fixed points $\{(o,[y, \omega]) \in \mathscr{A} \mid y=0,1 / 2, \omega / 2$ or $(1+\omega) / 2\}$ and by suitable coordinates in their neighborhoods, $g$ and $\phi_{1} \times \psi_{1}$ are expressed as follows:

$$
\begin{aligned}
\mathrm{g}:(x, y, z, w) & \mapsto(-x,-y,-z,-w), \\
\phi_{1} \times \psi_{1}:(x, y, z, w) & \mapsto(x y, w) .
\end{aligned}
$$

Thus some neighborhood of the singular points of $\mathscr{A} / \mathrm{g}^{Z}$ is isomorphic to $N_{2} \times\left(\mathscr{S} / \Gamma_{o}(4)\right)$. Let $\mathscr{S}$ be the non-singular model of $\mathscr{S} / \mathfrak{g}^{Z}$ as in (II) of Section 2, and let $\pi_{\mathrm{i}}: \mathscr{S} \rightarrow \Delta_{\infty} \times\left(\mathscr{S} / \Gamma_{o}(4)\right)$ be the holomorphic map induced by $\phi_{1} \times \psi_{1}$. Then $\pi_{i}^{-1}(0,[\omega])$ consists of five components crossing normally. A component $V$ is isomorphic to a non-singular model of $\boldsymbol{P}^{1} \times E(\omega) / \bar{g}^{z}$, where $\bar{g}:(\eta,[y]) \mapsto(\eta,[y])$, thus is an elliptic surface over $\boldsymbol{P}^{1}$ with two singular fibers of type $I_{1}^{*}$. The other four components are isomorphic to $\boldsymbol{P}^{2}$. They intersect as in Figure (1-i). In particular, $V$ intersects with itself at the points $[\infty, y]$ and $[0, y]$, where $[\eta, y]$ denotes the image in $V$ of $(\eta,[y]) \in \boldsymbol{P}^{1} \times E(\omega)$. Let

$$
\begin{aligned}
& \mathfrak{p}: \Delta_{\infty} \times\left(\mathscr{S}_{\mathcal{E}} / \Gamma_{o}(4)\right) \ni(s,[\omega]) \mapsto[1, s, 0, \omega] \in \mathscr{S}, \\
& \mathfrak{p}: \Delta_{\infty} \times\left(\mathfrak{K}_{\mathcal{C}} / \Gamma_{o}(4)\right) \ni(s,[\omega]) \mapsto\left[\sigma_{4}, s, 0, \omega\right] \in \mathscr{S}, \\
& \mathfrak{q}: \Delta_{\infty} \times\left(\mathscr{K}_{\mathcal{E}} / \Gamma_{o}(4)\right) \ni(s,[\omega]) \mapsto[1, s, 1 / 4, \omega] \in \mathscr{S},
\end{aligned}
$$

where $[v, s, y, \omega]$ denotes the image in $\mathscr{S}$ of $([v, s],[y, \omega]) \in \mathscr{E}_{1} \times \mathscr{B}_{1}$. Then $\mathfrak{p}(0,[\omega]), \mathfrak{p}(0,[\omega])$ and $\mathfrak{q}(0,[\omega])$ are the points $[1,0],\left[\sigma_{4}, 0\right]$ and $[1$, 1/4]) of $V$, respectively.
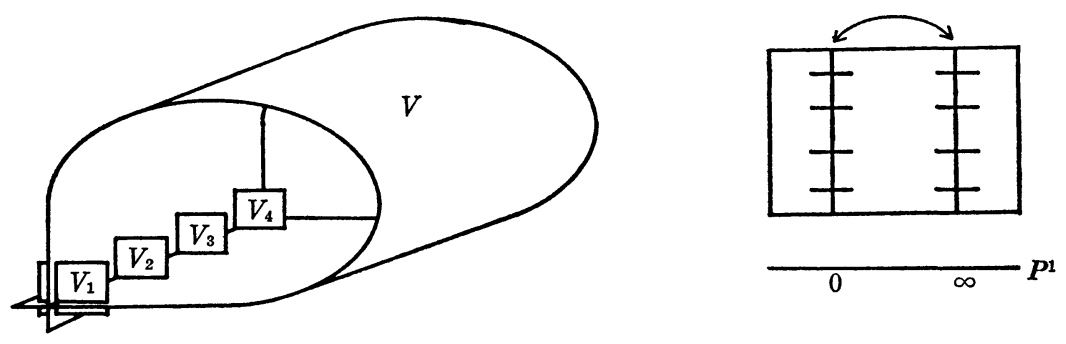

FIGURE 1-i

(1-ii) Over $\Delta_{0} \times\left(\mathscr{S}_{\mathrm{C}} / \Gamma_{\mathrm{o}}(4)\right)$.

Let $\mathscr{A}=\mathscr{E}_{4} \times \mathscr{B}_{1}$ and

$$
\mathrm{g}: \mathscr{A} \ni(p,[y, \omega]) \mapsto\left(\beta^{2} p,[-y, \omega]\right) \in \mathscr{A} \text {. }
$$


Then $\mathfrak{g}$ has no fixed point, hence $\mathscr{S}=\mathscr{A} / \mathfrak{g}^{Z}$ is non-singular. Let $\pi_{\mathrm{ii}}$ be the holomorphic map from $\mathscr{S}$ to $\Delta_{0} \times\left(\mathscr{S}_{\mathrm{S}} / \Gamma_{0}(4)\right)$ induced by $\phi_{4} \times \psi_{1}$. Then $\pi_{\mathrm{ii}}^{-1}(0,[\omega])$ is the analytic space consisting of two components $V_{1}$ and $V_{2}$ both isomorphic to $\boldsymbol{P}^{1} \times E(\omega)$, which intersect in the following way: The points $(0,[y])$ and $(\infty,[y])$ of $V_{1}$ meet the points $(\infty,[y])$ and $(0,[-y])$, respectively. Let $\mathfrak{b}, \mathfrak{q}$ be the sections of $\pi_{\mathrm{ii}}$ defined in the same way as in (1-i), and let

$$
\mathfrak{p}: \Delta_{0} \times\left(\mathscr{S}_{\mathfrak{g}} / \Gamma_{o}(4)\right) \in(s,[\omega]) \mapsto[s, s, 0, \omega] \in \mathscr{S} .
$$

Then $\mathfrak{D}(0,[\omega]), \mathfrak{q}(0,[\omega])$ are the points $(1,[0]),(1,[1 / 4])$ of $V_{1}$, respectively, and $\mathfrak{p}(0,[\omega])$ is the point $(1,[0])$ of $V_{2}$.
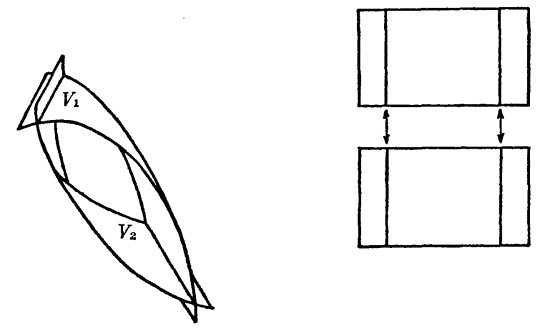

Figure 1-ii

(1-iii) Over $\Delta_{1 / 2} \times\left(\mathfrak{S} / \Gamma_{o}(4)\right)$.

Let $\mathscr{S}$ be the same manifold as in (1-i), and $\pi_{i 1 i}$ be the holomorphic map from $\mathscr{S}$ to $\Delta_{0} \times\left(\mathscr{S}_{\mathcal{C}} / \Gamma_{0}(4)\right)$ induced by $\dot{\phi}_{1} \times \psi_{1}$. Let $\mathfrak{p}, \mathfrak{q}$ be the sections of $\pi_{\mathrm{iii}}$ defined in the same way as in (1-i), and let

$$
\mathfrak{p}: \Delta_{1 / 2} \times\left(\mathfrak{S}_{\mathcal{E}} / \Gamma_{0}(4)\right) \ni(s,[\omega]) \mapsto\left[\sigma_{4} s^{1 / 2}, s, 0, \omega\right] \in \mathscr{S} .
$$

Then clearly $\pi_{\mathrm{iii}}^{-1}(0,[\omega])(\mathfrak{p}(0,[\omega]), \mathfrak{q}(0,[\omega]))$ is isomorphic to $\pi_{\mathrm{i}}^{-1}(0,[\omega])(\mathfrak{p}(0$, $[\omega]), \mathfrak{q}(0,[\omega]))$ in $(1-\mathrm{i})$, and $\mathfrak{p}(0,[\omega])$ is a point of a component isomorphic to $\boldsymbol{P}^{2}$.

(1-iv) Over $\left(\mathscr{S}_{\mathcal{C}} / \Gamma_{0}(4)\right) \times \widetilde{J}_{\infty}$.

Let $\mathscr{A}=\mathscr{P}_{1} \times \mathscr{E}_{2}$ and let

$$
\mathrm{g}: \mathscr{A} \ni([x, \tau], p) \mapsto([x+1 / 2, \tau], \gamma p) \in \mathscr{A} \text {. }
$$

Then $\mathscr{S}=\mathscr{A} / \mathfrak{g}^{Z}$ is non-singular, since $\mathrm{g}$ has no fixed point. Let $\pi_{\mathrm{iv}}: \mathscr{S} \rightarrow\left(\mathscr{S}_{\mathrm{C}} / \Gamma_{\mathrm{o}}(4)\right) \times \widetilde{\Delta}_{\infty}$ be the holomorphic map induced by $\psi_{1} \times \phi_{2}$. Let

$$
\begin{aligned}
& \mathfrak{p}:\left(\mathfrak{S}_{\mathcal{C}} / \Gamma_{o}(4)\right) \times \widetilde{\Delta}_{\infty} \ni([\tau], t) \mapsto[0, \tau, 1, t] \in \mathscr{S}, \\
& \mathfrak{p}:\left(\mathfrak{S}_{\mathcal{C}} / \Gamma_{o}(4)\right) \times \widetilde{\Delta}_{\infty} \ni([\tau], t) \mapsto[1 / 4, \tau, 1, t] \in \mathscr{S}, \\
& \mathfrak{q}:\left(\mathfrak{S}_{\mathcal{C}} / \Gamma_{o}(4)\right) \times \widetilde{\Delta}_{\infty} \ni([\tau], t) \mapsto\left[0, \tau, \sigma_{4}, t\right] \in \mathscr{S} .
\end{aligned}
$$

Then $\pi_{\mathrm{iv}}^{-1}([\tau], 0)$ is the analytic space consisting of two components $V_{1}$ and $V_{2}$ both isomorphic to $E(\tau) \times \boldsymbol{P}^{1} / \bar{g}^{z}$, with $\bar{g}:([x], \eta) \mapsto\left([x+1 / 2], \eta^{-1}\right)$ 
meeting normally at the points $[x, 0] \in V_{1}$ and $[x, \infty] \in V_{2} . \quad \mathfrak{D}([\tau], 0), \mathfrak{p}([\tau]$, 0 ) and $\mathfrak{q}([\tau], 0)$ are the points $[0,1],[1 / 4,1]$ and $\left[0, \sigma_{4}\right]$ of $V_{1}$, respectively. $(1-\mathrm{v})$ Over $\left(\mathfrak{S} / \Gamma_{o}(4)\right) \times \Delta_{0}$.

Let $\mathscr{A}=\mathscr{B}_{1} \times \mathscr{E}_{4}$ and $\mathfrak{g}$ be the automorphism of defined in the same way as in (1-iv). Then $\mathscr{S}=\mathscr{A} / \mathfrak{g}^{z}$ is non-singular. Let $\pi_{\mathrm{v}}: \mathscr{S} \rightarrow\left(\mathscr{S} / \Gamma_{o}(4)\right) \times \Delta_{0}$ be the holomorphic map induced by $\psi_{1} \times \dot{\phi}_{4}$. Then $\pi_{\mathrm{v}}^{-1}([\tau], 0)$ is the analytic space consisting of three components: $V_{1}, V_{2}$ both isomorphic to those of $\pi_{\mathrm{iv}}^{-1}([\tau], 0)$ in (1-iv) and $V_{3}$ isomorphic to $E(\tau) \times \boldsymbol{P}^{1}$ with the points $[x, \infty]$ of $V_{1}$ and $[x, 0]$ of $V_{2}$ meeting the points $([x], 0)$ and $([x], \infty)$, respectively. Let $\mathfrak{D}, \mathfrak{p}$ be the sections of $\pi_{\mathrm{v}}$ defined in the same way as in (1-iv), and let

$$
\mathrm{q}:\left(\mathfrak{S}_{\mathcal{C}} / \Gamma_{o}(4)\right) \times \Delta_{0} \ni([\tau], t) \mapsto[0, \tau, t, t] \in \mathscr{S} .
$$

Then $\mathfrak{q}([\tau], 0)$ is the point $([0], 1)$ of $V_{3}$.

(1-vi) Over $\left(\mathfrak{S} / \Gamma_{o}(4)\right) \times \widetilde{\Delta}_{1 / 2}$.

Let $\mathscr{S}$ be the same manifold as in (1-iv), and let $\pi_{\mathrm{vi}}: \mathscr{S} \rightarrow$ $\left(\mathscr{S} / \Gamma_{o}(4)\right) \times \widetilde{\Delta}_{1 / 2}$ be the holomorphic map induced by $\psi_{1} \times \phi_{2}$. Let $\mathfrak{D}, \mathfrak{p}$ be the sections of $\pi_{\mathrm{vi}}$ defined in the same way as in (1-iv), and let

$$
\mathfrak{q}:\left(\mathscr{S} / \Gamma_{o}(4)\right) \times{\widetilde{J_{1 / 2}}} \ni([\tau], t) \mapsto\left[0, \tau, \sigma_{4} t, t\right] \in \mathscr{S} .
$$

Then clearly $\pi_{\mathrm{vi}}^{-1}([\tau], 0)(\mathfrak{p}([\tau], 0), \mathfrak{p}([\tau], 0))$ is isomorphic to $\pi_{\mathrm{iv}}^{-1}([\tau], 0)(\mathfrak{p}([\tau], 0)$, $\mathfrak{p}([\tau], 0))$ in $(1-\mathrm{iv})$, and $\mathrm{q}([\tau], 0)$ is the point $\left[0, \sigma_{4}\right]$ of $V_{2}$.

(1-vii) Over $\Delta_{\infty} \times \tilde{\Delta}_{\infty}$.

Let $\mathscr{A}=\mathscr{A}_{1 \mid \Delta_{\infty} \times \tilde{J}_{\infty}}$ and $\mathfrak{g}=\left[\theta \circ \mu_{*}\right]$. Then $\mathfrak{g}$ has fixed points $\{[0, w$, $s, t] \in \mathscr{R} \mid w= \pm 1, \pm t\}$ on $\mathscr{A}$, and some neighborhood of singular points of $\mathscr{\Omega} / \mathrm{g}^{z}$ is isomorphic to $N_{2} \times \widetilde{\Delta}_{\infty}$. Thus we can obtain the non-singular model $\mathscr{S}$ of $\mathscr{A} / \mathfrak{g}^{Z}$. Let $\pi_{\mathrm{vi}}: \mathscr{S} \rightarrow \Delta_{\infty} \times \widetilde{\Delta}_{\infty}$ be induced by $\widetilde{\sigma}_{1}$. Then $\pi_{\mathrm{vii}}^{-1}(0,0)$ consists of two components $V_{1}, V_{2}$ isomorphic to the non-singular model of $\boldsymbol{P}^{1} \times \boldsymbol{P}^{1} / \bar{g}^{z}$, where $\bar{g}:(\eta, \xi) \mapsto\left(-\eta, \xi^{-1}\right)$, and four components $V_{3}, V_{4}, V_{5}, V_{6}$ isomorphic to $\boldsymbol{P}^{2}$. They intersect as in Figure 1-vii. In particular, the points [ $\eta, 0]$ of $V_{1}$ meet the points $[\eta, \infty]$ of $V_{2}$, the points $[0, \xi]$ of $V_{1}\left(\operatorname{resp} . V_{2}\right)$ meet the points $[\infty, \xi]$ of $V_{1}$ (resp. $V_{2}$ ). Let

$$
\begin{aligned}
& \mathfrak{D}: \Delta_{\infty} \times \tilde{\Delta}_{\infty} \ni(s, t) \mapsto[1,1, s, t] \in \mathscr{S}, \\
& \mathfrak{p}: \Delta_{\infty} \times \widetilde{\Delta}_{\infty} \ni(s, t) \mapsto\left[\sigma_{4}, 1, s, t\right] \in \mathscr{S}, \\
& \mathfrak{q}: \Delta_{\infty} \times \widetilde{\Delta}_{\infty} \ni(s, t) \mapsto\left[1, \sigma_{4}, s, t\right] \in \mathscr{S} .
\end{aligned}
$$

Then $\mathfrak{p}(0,0), \mathfrak{p}(0,0)$ and $\mathfrak{q}(0,0)$ are the points $[1,1],\left[\sigma_{4}, 1\right]$ and $\left[1, \sigma_{4}\right]$ of $V_{1}$, respectively. 

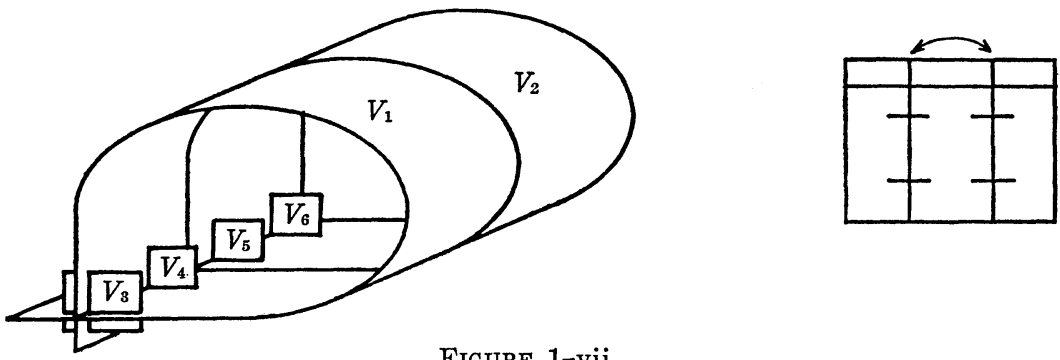

(1-viii) Over $\Delta_{0} \times \widetilde{\Delta}_{\infty}$.

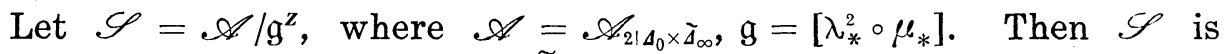
non-singular. Let $\pi_{\mathrm{vii} i}: \mathscr{S} \rightarrow \Delta_{0} \times \tilde{\Delta}_{\infty}$ be induced by $\widetilde{\sigma}_{2}$. Then $\pi_{\mathrm{vii}}^{-1}(0,0)$ consists of four components $V_{1}, V_{2}, V_{3}$ and $V_{4}$ isomorphic to $\boldsymbol{P}^{1} \times \boldsymbol{P}^{1}$, and intersecting in the following way: The points $(0, \xi),(\infty, \xi)$ of $V_{1}$ (resp. $\left.V_{3}\right)$ meet the points $\left(\infty, \xi^{-1}\right),(0, \xi)$ of $V_{2}$ (resp. $\left.V_{4}\right)$, and the points $(\eta, \infty),(\eta, 0)$ of $V_{1}\left(\operatorname{resp} . V_{2}\right)$ meet the points $(\eta, \infty),(\eta, 0)$ of $V_{3}\left(\operatorname{resp} . V_{4}\right)$. Let $\mathfrak{v}, \mathfrak{q}$ be the sections of $\pi_{v i i i}$ defined in the same way as in (1-xii), and let

$$
\mathfrak{p}: \Delta_{0} \times \widetilde{\Delta}_{\infty} \ni(s, t) \mapsto[s, 1, s, t] \in \mathscr{S} .
$$

Then $\mathfrak{p}(0,0), \mathfrak{q}(0,0)$ are the points $(1,1),\left(1, \sigma_{4}\right)$ of $V_{1}$ and $\mathfrak{p}(0,0)$ is the point $(1,1)$ of $V_{2}$.

(1-ix) Over $\Delta_{1 / 2} \times \tilde{\Delta}_{\infty}$.

Let $\mathscr{S}$ be the same manifold as in (1-vii), and let $\pi_{\mathrm{ix}}: \mathscr{S} \rightarrow \Delta_{1 / 2} \times \tilde{\Delta}_{\infty}$ be induced by $\widetilde{\sigma}_{1}$. Let $\mathfrak{D}, \mathfrak{q}$ be the sections of $\pi_{\mathrm{ix}}$ defined in the same way as in (1-vii), and let

$$
\mathfrak{p}: \Delta_{1 / 2} \times \Delta_{\infty} \ni(s, t) \mapsto\left[\sigma_{4} s^{1 / 2}, 1, s, t\right] \in \mathscr{S} .
$$

Then clearly $\pi_{\mathrm{ix}}^{-1}(0,0)(\mathfrak{D}(0,0), \mathfrak{q}(0,0))$ is isomorphic to $\pi_{\mathrm{vii}}^{-1}(0,0)(\mathfrak{D}(0,0), \mathfrak{q}(0,0))$ in (1-vii), and $\mathfrak{p}(0,0)$ is a point of $V_{3}$.

(1-x) Over $\Delta_{\infty} \times \Delta_{0}$.

Let $\mathscr{A}=\mathscr{A}_{3 \mid \Delta_{\infty} \times \Delta_{0}}$ and $\mathfrak{g}=\left[\theta \circ \mu_{*}\right]$. Then we can obtain the nonsingular model of $\mathscr{A} / \mathfrak{g}^{z}$ in the same way as in (1-vii). Let $\pi_{x}: \mathscr{S} \rightarrow$ $\Delta_{\infty} \times \Delta_{0}$ be the holomorphic map induced by $\varpi_{3}$. Then $\pi_{x}^{-1}(0,0)$ consists of two components $V_{1}, V_{2}$ isomorphic to those in (1-vii) a component $V_{3}$ isomorphic to $\boldsymbol{P}^{1} \times \boldsymbol{P}^{1}$, four components $V_{4}, V_{5}, V_{6}$ and $V_{7}$ isomorphic to $P^{2}$ intersecting as in Figure 1-x. In particular, the points $[0, \xi]$ of $V_{1}$ (resp. $\left.V_{2}\right)$ meet the points $[\infty, \xi]$ of $V_{1}\left(\operatorname{resp} . V_{2}\right)$, the points $[\eta, \infty]$ of $V_{1}$ and $[\eta, 0]$ of $\mathrm{V}_{2}$ meet the points $(\eta, 0)$ and $(\eta, \infty)$ of $V_{3}$, respectively. Let $\mathfrak{D}, \mathfrak{p}$ be the sections of $\pi_{x}$ defined in the same way as in (1-vii), and let 

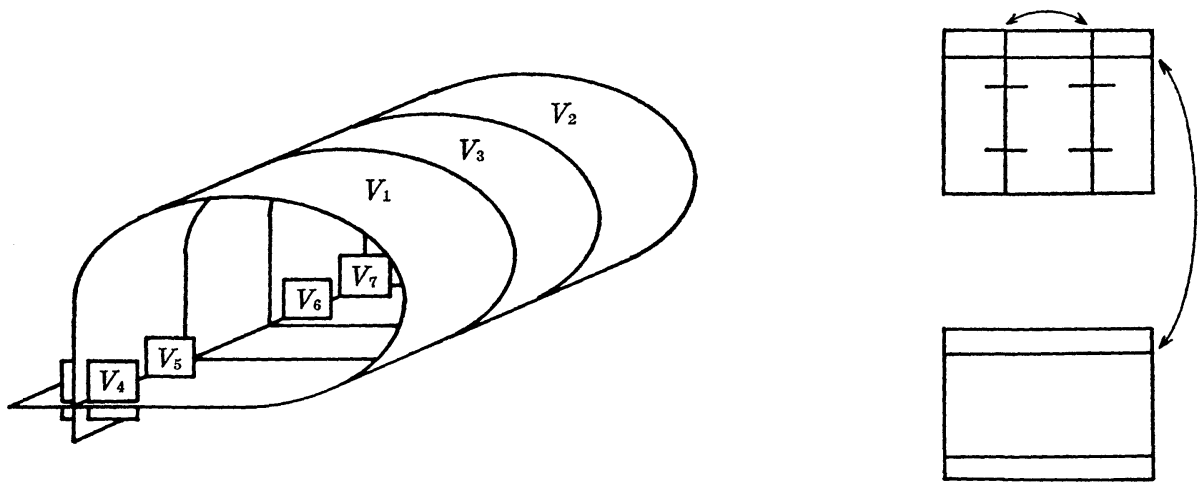

Figure 1-x

$$
\mathfrak{q}: \Delta_{\infty} \times \Delta_{0} \ni(s, t) \mapsto[1, t, s, t] \in \mathscr{S} .
$$

Then $\mathfrak{o}(0,0), \mathfrak{p}(0,0)$ are the points $[1,1],\left[\sigma_{4}, 1\right]$ of $V_{1}$, respectively, and $\mathfrak{q}(0,0)$ is the point $(1,1)$ of $V_{3}$.

(1-xi) Over $\Delta_{0} \times \Delta_{0}$.

Let $\mathscr{S}=\mathscr{A} / \mathfrak{g}^{Z}$, where $\mathscr{A}=\mathscr{A}_{4 \mid \Delta_{0} \times \Delta_{0}}, \mathfrak{g}=\left[\lambda_{*}^{2} \circ \mu_{*}\right]$. Then $\mathscr{S}$ is non-singular. Let $\pi_{\mathrm{xi}}: \mathscr{S} \rightarrow \Delta_{0} \times \Delta_{0}$ be the holomorphic map induced by $\widetilde{\sigma}_{4}$. Then $\pi_{\mathrm{xi}_{1}}^{-1}(0,0)$ consists of eight components isomorphic to $\boldsymbol{P}^{1} \times \boldsymbol{P}^{1}$ intersecting in the following way: The points $(\infty, \xi)$ of $V_{1}, V_{2}, V_{3}, V_{4}$, $V_{5}, V_{6}, V_{7}, V_{8}$ meet the point $(0, \xi)$ of $V_{5}, V_{6}, V_{7}, V_{8},\left(0, \xi^{-1}\right)$ of $V_{4}, V_{1}, V_{3}, V_{2}$, respectively. The points $(\eta, \infty)$ of $V_{1}, V_{2}, V_{3}, V_{4}, V_{5}, V_{6}, V_{7}, V_{8}$ meet the points $(\eta, 0)$ of $V_{2}, V_{3}, V_{4}, V_{1}, V_{6}, V_{7}, V_{8}, V_{5}$, respectively. Let $\mathfrak{0}, \mathfrak{p}$ and $q$ be the sections of $\pi_{x i}$ defined in the same way as in (1-vii), (1-viii) and $(1-x)$, respectively. Then $\mathfrak{o}(0,0), \mathfrak{p}(0,0)$ and $\mathfrak{q}(0,0)$ are the points $(1,1)$ of $V_{1}, V_{5}$ and $V_{2}$, respectively.

(1-xii) Over $\Delta_{1 / 2} \times \Delta_{0}$.

Let $\mathscr{S}$ be the same manifold as in (1-x), and let $\pi_{\mathrm{xi} i}: \mathscr{S} \rightarrow \Delta_{1 / 2} \times \Delta_{0}$ be induced by $\widetilde{\sigma}_{3}$. Let $\mathfrak{o}, \mathfrak{p}$ and $\mathfrak{q}$ be the sections of $\pi_{x i 1}$ defined in the same way as in (1-vii), (1-ix) and (1-x), respectively. Then clearly $\pi_{\mathrm{xii}}^{-1}(0,0)(\mathfrak{D}(0,0), \mathfrak{q}(0,0))$ is isomorphic to $\pi_{\mathrm{x}}^{-1}(0,0)(\mathfrak{D}(0,0), q(0,0))$ in $(1-\mathrm{x})$, and $\mathfrak{p}(0,0)$ is a point of $V_{4}$.

(1-xiii) Over $\Delta_{\infty} \times \widetilde{\Delta}_{1 / 2}$.

Let $\mathscr{S}$ be the same manifold as in (1-vii), and let $\pi_{\mathrm{xiii}}: \mathscr{S} \rightarrow \Delta_{\infty} \times \widetilde{\Delta}_{1 / 2}$ be the holomorphic map induced by $\widetilde{\sigma}_{1}$. Let $\mathfrak{D}$ and $\mathfrak{p}$ be the sections of $\pi_{x i i i}$ defined in the same way as in (1-vii), and let

$$
\mathfrak{q}: \Delta_{\infty} \times \widetilde{\Delta}_{1 / 2} \ni(s, t) \mapsto\left[1, \sigma_{4} t, s, t\right] \in \mathscr{S} .
$$

Then clearly $\pi_{\mathrm{x} i \mathrm{ii}}^{-1}(0,0)(\mathfrak{p}(0,0), \mathfrak{p}(0,0))$ is isomorphic to $\pi_{\mathrm{v} \mathbf{i}}^{-1}(0,0)(\mathfrak{p}(0,0)$, 
$\mathfrak{p}(0,0))$ in (1-vii), and $\mathfrak{q}(0,0)$ is the point $\left[1, \sigma_{4}\right]$ of $V_{2}$.

(1-xiv) Over $\Delta_{0} \times \tilde{\Delta}_{1 / 2}$.

Let $\mathscr{S}$ be the same manifold as in (1-viii), and let $\pi_{x i v}: \mathscr{S} \rightarrow \Delta_{0} \times \widetilde{A}_{1 / 2}$ be the holomorphic map induced by $\widetilde{\sigma}_{2}$. Let $\mathfrak{o}, \mathfrak{p}$ and $\mathfrak{q}$ be the sections of $\pi_{x \mathrm{x} v}$ defined in the same way as in (1-vii), (1-viii) and (1-xiii), respectively. Then clearly $\pi_{\mathrm{xiv}}^{-1}(0,0)(\mathfrak{p}(0,0), \mathfrak{p}(0,0))$ is isomorphic to $\pi_{\mathrm{viii}}^{-1}(0,0)$ $(\mathfrak{p}(0,0), \mathfrak{p}(0,0))$ in (1-viii), and $\mathfrak{q}(0,0)$ is the point $\left(1, \sigma_{4}\right)$ of $V_{3}$. (1-xv) Over $\Delta_{1 / 2} \times \widetilde{\Delta}_{1 / 2}$.

Let $\mathscr{S}$ be the same manifold as in (1-ix), and let $\pi_{\mathrm{xv}}: \mathscr{S} \rightarrow \Delta_{1 / 2} \times{\widetilde{\Delta_{1 / 2}}}$ be the holomorphic map induced by $\widetilde{\varpi}_{1}$. Let $\mathfrak{p}, \mathfrak{p}$ and $\mathfrak{q}$ be the sections of $\pi_{\mathrm{x} v}$ defined in the same way as in (1-vii), (1-ix) and (1-xiii), respectively. Then clearly $\pi_{\mathrm{xv}}^{-1}(0,0)(\mathrm{p}(0,0), \mathrm{q}(0,0))$ is isomorphic to $\pi_{\mathrm{xii1}}^{-1}(0,0)(\mathrm{p}(0$ $0), \mathfrak{q}(0,0))$ in (1-xiii), and $\mathfrak{p}(0,0)$ is a point of $V_{3}$.

(2-i) Over $\Delta_{\infty} \times\left(\mathfrak{S} / \Gamma_{0}(4)\right)$.

Let $\mathscr{A}=\mathscr{E}_{2} \times \mathscr{B}_{1} / h^{z}$, where $h:(p,[y, \omega]) \mapsto(\beta p,[y+1 / 2, \omega])$. Then we can obtain the family $\pi_{\mathrm{i}}: \mathscr{S} \rightarrow \Delta_{\infty} \times\left(\mathscr{H}_{\mathcal{C}} / \Gamma_{o}(4)\right)$ together with sections $\mathfrak{v}, \mathfrak{q}$ in the same way as in (1-i). Let $\mathfrak{p}$ be the quasi-section of $\pi_{\mathrm{i}}$ defined by

$$
\mathfrak{p}:(s,[\omega]) \mapsto\left\{\left[\sigma_{4}, s, y, \omega\right] \mid y=0,1 / 2\right\} .
$$

Then $\pi_{\mathrm{i}}^{-1}(0,[\omega])$ consists of the components isomorphic to those of $\pi_{\mathrm{i}}^{-1}(0,[\omega])$ in $(1-\mathrm{i})$. But the points $[\infty, y]$ of $V$ meet the points $[0, y+$ 1/2] of itself. $\mathfrak{p}(0,[\omega])$ is the pair of the points $\left[\sigma_{4}, 0\right],\left[\sigma_{4}, 1 / 2\right]$ of $V$.

(2-ii) Over $\Delta_{0} \times\left(\mathscr{S}_{\mathrm{C}} / \Gamma_{\mathrm{o}}(4)\right)$.

Let $\mathscr{A}=\mathscr{E}_{2} \times \mathscr{B}_{1} / h^{z}$, where $h:(p,[y, \omega]) \mapsto\left(\alpha_{2} p,[y+1 / 2, \omega]\right)$. Then we obtain the family $\pi_{\mathrm{ii}}: \mathscr{S} \rightarrow \Delta_{0} \times\left(\mathfrak{\mathcal { E }} / \Gamma_{0}(4)\right)$ together with sections $\mathrm{o}$ and $\mathfrak{q}$ in the same way as in (1-ii). Let $\mathfrak{p}$ be the quasi-section of $\pi_{\mathrm{ii}}$ defined by

$$
\mathfrak{p}:(s,[\omega]) \mapsto\left\{\left[ \pm s^{1 / 2}, s, 0, \omega\right]\right\} .
$$

Then $\pi_{\mathrm{ii}}^{-1}(0,[\omega])$ consists of the component $V$ whose normalization is isomorphic to $\boldsymbol{P}^{1} \times E(\omega) / \bar{g}^{z}$, where $\bar{g}:(\eta,[y]) \mapsto(-\eta,[y+1 / 2])$, thus an elliptic surface with two double fibers. $\mathfrak{p}(0,[\omega])$ is a point on the double
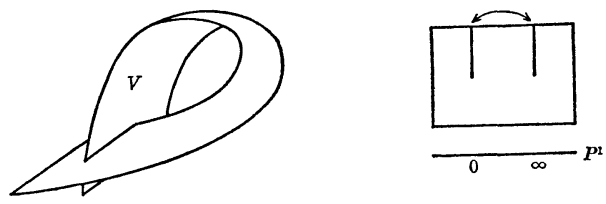

Figure 2-ii 
curve of $V$.

(2-iii) Over $\Delta_{1 / 2} \times\left(\mathfrak{S} / \Gamma_{o}(4)\right)$.

Let $\mathscr{A}$ be the same manifold as in (2-i), and let $\mathrm{g}:[p, y, \omega] \mapsto$ $\left[\alpha_{2} \beta p,-y, \omega\right]$. Then we obtain the family $\pi_{\mathrm{iij}}: \mathscr{S} \rightarrow \Delta_{1 / 2} \times\left(\mathcal{S}_{\mathcal{C}} / \Gamma_{o}(4)\right)$ together with sections $\mathfrak{D}, \mathfrak{q}$. Let $\mathfrak{p}$ be the quasi-section of $\pi_{\mathrm{iii}}$ defined by

$$
\mathfrak{p}:(s,[\omega]) \mapsto\left\{\left[\sigma_{4} s^{1 / 2}, s, y, \omega\right] \mid y=0,1 / 2\right\} .
$$

Then $\pi_{\mathrm{ii}}^{-1}(0,[\omega])$ is isomorphic to $\pi_{\mathrm{i}}^{-1}(0,[\omega])$ in $(2-\mathrm{i}), \mathfrak{o}(0,[\omega]), \mathfrak{q}([0,[\omega])$ are the points $[1,1 / 4],[1,1 / 2]$ of $V_{1}$, respectively, and $\mathfrak{p}(0,[\omega])$ is a pair of points one on $V_{2}$ and the other on $V_{4}$.

(2-iv) Over $\left(\mathfrak{\mathcal { C }} / \Gamma_{o}(4)\right) \times \widetilde{\Delta}_{\infty}$.

Let $\mathscr{A}=\mathscr{B}_{2} \times \mathscr{E}_{2} / h^{z}$, where $h:([x, \tau], p) \mapsto\left([x+\tau, \tau], \alpha_{2} p\right)$. Then we obtain the family $\pi_{\mathrm{iv}}: \mathscr{S} \rightarrow\left(\mathfrak{S} / \Gamma_{0}(4)\right) \times \widetilde{\Delta}_{\infty}$ together with the sections $\mathrm{D}, \mathrm{q}$ in the same way as in (1-iv). Let $\mathfrak{p}$ be the quasi-section of $\pi_{\mathrm{iv}}$ defined by

$$
\mathfrak{p}:([\tau], t) \mapsto\{[x, \tau, 1, t] \mid x=1 / 4,1 / 4+\tau\} .
$$

Then $\pi_{\mathrm{iv}}^{-1}([\tau], 0)$ consists of two components $V_{1}$ and $V_{2}$ isomorphic to $E(2 \tau) \times \boldsymbol{P}^{1} / \bar{h}^{Z} \times \bar{g}^{Z}$, where $\bar{h}:([x], \eta) \mapsto([x+\tau],-\eta), \bar{g}:([x], \eta) \mapsto([x+1 / 2]$, $\left.\eta^{-1}\right)$, with the points $[x, \infty]$ and $[x, 0]$ of $V_{1}$ meeting the points $[x, 0]$ and $[x, \infty]$ of $V_{2}$, respectively. $\mathfrak{p}([\tau], 0)$ is the pair of the points $[1 / 4,1]$ and $[1 / 4+\tau, 1]$ of $V_{1}$.

$(2-\mathrm{v})$ Over $\left(\mathfrak{S} / \Gamma_{0}(4)\right) \times \Delta_{0}$.

Let $\mathscr{A}=\mathscr{B}_{2} \times \mathscr{E}_{4} / h^{z}$, where $h:([x, \tau], p) \mapsto\left([x+\tau, \tau], \beta^{2} p\right)$. Then we obtain the family $\pi_{\mathrm{v}}: \mathscr{S} \rightarrow\left(\mathscr{C} / \Gamma_{\mathrm{o}}(4)\right) \times \Delta_{0}$ together with the sections $\mathfrak{b}$ and $\mathfrak{q}$, in the same way as in $(1-v)$. Let $\mathfrak{p}$ be the quasi-section of $\pi_{\mathrm{v}}$ defined in the same way as in $(2-i v)$. Then $\pi_{v}^{-1}([\tau], 0)$ consists of components: $V_{1}$ isomorphic to that of $\pi_{\mathrm{iv}}^{-1}([\tau], 0)$ in (1-iv), and $V_{2}$ isomorphic to $E(2 \tau) \times \boldsymbol{P}^{1} / \bar{g}^{z}$, where $\bar{g}:([x], \xi) \mapsto\left([x+\tau+1 / 2], \xi^{-1}\right)$. The points $[x, \infty]$ of $V_{1}$ meet the points $[x, 0]$ of $V_{2}$. And $\mathfrak{o}([\tau], 0), \mathfrak{q}([\tau], 0)$ are the points $[0,1]$ of $V_{1}, V_{2}$, respectively, $\mathfrak{p}([\tau], 0)$ is the pair of the points $[1 / 4,1]$ and $[1 / 4+\tau, 1]$ of $V_{1}$.

(2-vi) Over $\left(\mathfrak{S} / \Gamma_{o}(4)\right) \times \tilde{\Delta}_{1 / 2}$.

Let $\mathscr{S}$ be the same manifold as in (2-iv), and let $\pi_{\mathrm{vi}}: \mathscr{S} \rightarrow\left(\mathscr{S} / \Gamma_{o}(4)\right) \times$ $\Delta_{1 / 2}$ be the holomorphic map induced by $\psi_{2} \times \phi_{2}$. Let $\mathfrak{o}, \mathfrak{q}$ and $\mathfrak{p}$ be the sections and the quasi-section of $\pi_{v i}$ defined in the same way as in (1-vi) and (2-iv), respectively. Then clearly $\pi_{\mathrm{v} i}^{-1}([\tau], 0)(\mathfrak{D}([\tau], 0), \mathfrak{p}([\tau], 0))$ is isomorphic to $\pi_{\mathrm{iv}}^{-1}([\tau], 0)(\mathfrak{D}([\tau], 0), \mathfrak{p}([\tau], 0))$ in $(2-\mathrm{iv})$, and $q([\tau], 0)$ is the point $\left[0, \sigma_{4}\right]$ of $V_{2}$.

(2-vii) Over $\Delta_{\infty} \times \tilde{\Delta}_{\infty}$. 


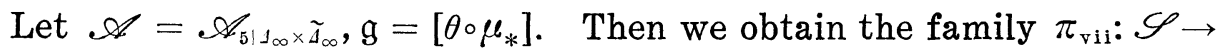
$\Delta_{\infty} \times \widetilde{\Delta}_{\infty}$ together with sections $\mathrm{D}$ and $\mathfrak{q}$ in the same way as in (1-vii). Let $\mathfrak{p}$ be the quasi-section defined by

$$
\mathfrak{p}:(s, t) \mapsto\left\{\left[\sigma_{4}, \pm 1, s, t\right]\right\} .
$$

Then $\pi_{\mathrm{vil}}^{-1}(0,0)$ consists of six components isomorphic to those of $\pi_{\mathrm{vii}}^{-1}(0,0)$ in (1-vii). The points $[0, \xi]$ of $V_{1}$ (resp. $V_{2}$ ) meet the points $[\infty, \xi]$ of $V_{1}$ (resp. $V_{2}$ ) and the points $[\eta, 0]$ of $V_{1}$ meet the points $[\eta, \infty]$ of $V_{2}$. $\mathfrak{p}(0,0)$ is the pair of the points $\left[\sigma_{4}, 1\right]$ and $\left[\sigma_{4},-1\right]$ of $V_{1}$.

(2-viii) Over $\Delta_{0} \times \widetilde{\Delta}_{\infty}$.

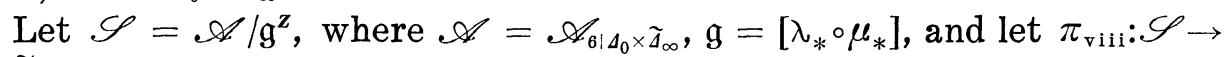
$\Delta_{0} \times \widetilde{\Delta}_{\infty}$ be the holomorphic map induced by $\widetilde{\sigma}_{6}$. Let $\mathfrak{p}$ be the quasisection of $\pi_{v i i i}$ defined by

$$
\mathfrak{p}:(s, t) \mapsto\left\{\left[ \pm s^{1 / 2}, 1, s, t\right]\right\} .
$$

Then $\mathscr{S}$ has two isolated singular points isomorphic to those of $\mathscr{A}_{6}$, and $\pi_{\mathrm{vii}}^{-1}(0,0)$ consists of two components whose normalization is isomorphic to $\boldsymbol{P}^{1} \times \boldsymbol{P}^{1} / \bar{h}^{\boldsymbol{z}}$, with $\bar{h}:(\eta, \xi) \mapsto(-\eta,-\xi)$. The points $[\eta, \infty]$ of $V_{1}$ (resp. $V_{2}$ ) meet the points [ $\left.\eta, 0\right]$ of $V_{2}$ (resp. $V_{1}$ ), and the points $[0, \xi]$ of $V_{1}$ (resp. $\left.V_{2}\right)$ meet the points $[\infty, \xi]$ of $V_{1}\left(\right.$ resp. $\left.V_{2}\right) . \mathfrak{p}(0,0)$ is a point on the double curve of $V_{1}$.

(2-ix) Over $\Delta_{1 / 2} \times \widetilde{\Delta}_{\infty}$.

Let $\mathscr{A}$ be the same manifold as in (2-vii), and let $\mathfrak{g}=\left[\theta \circ \lambda \circ \mu_{*}\right]$. Then we obtain the family $\pi_{\mathrm{ix}}: \mathscr{S} \rightarrow \Delta_{1 / 2} \times \Delta_{\infty}$ together with the sections $\mathfrak{o}$ and $\mathfrak{q}$ in the same way as in (2-vii). Let $\mathfrak{p}$ be the quasi-section of $\pi_{\mathrm{ix}}$ defined by

$$
\mathfrak{p}:(s, t) \mapsto\left\{\left[ \pm \sigma_{4} s^{1 / 2}, \sigma_{4}, s, t\right]\right\} .
$$

Then $\pi_{\mathrm{ix}}^{-1}(0,0)$ is isomorphic to $\pi_{\mathrm{vii}}^{-1}(0,0)$ in $(2$-vii), $\mathfrak{o}(0,0), \mathfrak{q}(0,0)$ are the points $\left[1, \sigma_{4}\right],[1,-1]$ of $V_{1}$, respectively, and $\mathfrak{p}(0,0)$ is a pair of points of $V_{3}$ and $V_{4}$.

(2-x) Over $\Delta_{\infty} \times \Delta_{0}$.

Let $\mathscr{A}=\mathscr{A}_{7 \mid \Delta_{\infty} \times \Delta_{0}}, \mathfrak{g}=\left[\theta \circ \mu_{*}\right]$. Then we obtain the family $\pi_{\mathrm{x}}: \mathscr{S} \rightarrow$ $\Delta_{\infty} \times \Delta_{0}$ together with the sections $\mathrm{D}$ and $\mathfrak{q}$ in the same way as in (1-x). Let $\mathfrak{p}$ be the quasi-section of $\pi$ defined by

$$
\mathfrak{p}:(s, t) \mapsto\left\{\left[\sigma_{4}, 1, s, t\right],\left[\sigma_{4}, t^{2}, s, t\right]\right\} .
$$

Then $\pi_{\mathrm{x}}^{-1}(0,0)$ consists of seven components isomorphic to those of $\pi_{x}^{-1}(0,0)$ in $(1-x)$, and intersecting as in Figure 2-x. In particular, the points $[0, \xi]$ of $V_{1}$ (resp. $[0, \xi]$ of $V_{2},[\eta, 0]$ of $V_{1},[\eta, 0]$ of $V_{2},(0, \xi)$ of $\left.V_{3}\right)$ 


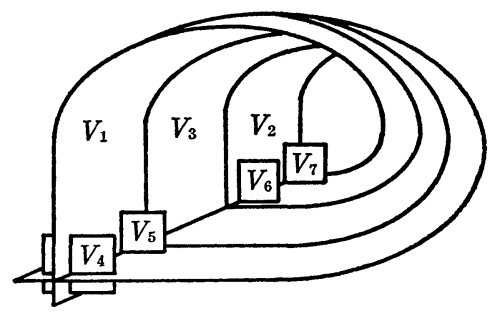

FIGURE 2-x

meet the points $[\infty, \xi]$ of $V_{2}$ (resp. $[\infty, \xi]$ of $V_{1},(\eta, 0)$ of $V_{3},(\eta, \infty)$ of $V_{3},(\infty, \xi)$ of $\left.V_{3}\right), \mathfrak{p}(0,0)$ is the pair of the points $\left[\sigma_{4}, 1\right]$ of $V_{1}$ and $\left(\sigma_{4}, 1\right)$ of $V_{3}$.

(2-xi) Over $\Delta_{0} \times \Delta_{0}$.

Let $\mathscr{A}=\mathscr{A}_{8 \mid \Delta_{0} \times \Delta_{0}}, \mathfrak{g}=\left[\lambda_{*} \circ \mu_{*}\right]$. Then we obtain the family $\pi_{\mathrm{xi}}: \mathscr{S} \rightarrow$ $\Delta_{0} \times \Delta_{0}$ together with sections $\mathfrak{o}$ and $\mathfrak{q}$ in the same way as in (1-xi). Let $\mathfrak{p}$ be the quasi-section of $\pi_{i x}$ defined in the same way as in (2-viii). Then $\pi_{\mathrm{xi}}^{-1}(0,0)$ consists of four components $V_{1}, V_{2}, V_{3}$ and $V_{4}$ isomorphic to $\boldsymbol{P}^{1} \times \boldsymbol{P}^{1}$, and intersect in the following way: The points $(\infty, \xi)$ of $V_{1}$ (resp. $\left.V_{2}\right)$ meet the points $(0, \xi)$ of $V_{3}$ (resp. $\left.V_{4}\right)$, the points $(0, \xi)$ of $V_{1}$ (resp. $\left.V_{2}\right)$ meet the points $\left(\infty, \xi^{-1}\right)$ of $V_{3}$ (resp. $\left.V_{4}\right)$, the points $(\eta, \infty)$ of $V_{1}$ (resp. $\left.V_{3}\right)$ meet the points $(\eta, 0)$ of $V_{2}$ (resp. $\left.V_{4}\right)$, and the points $(\eta, 0)$ of $V_{1}$ (resp. $\left.V_{3}\right)$ meet the points $(-\eta, \infty)$ of $V_{2}$ (resp. $\left.V_{4}\right)$. And $\mathfrak{p}(0,0)$ is the point obtained by identifing the point $(0,1)$ of $V_{1}$ with the point $(\infty, 1)$ of $V_{3}$.

(2-xii) Over $\Delta_{1 / 2} \times \Delta_{0}$.

Let $\mathscr{A}=\mathscr{A}_{7 \mid 1_{1 / 2} \times \Delta_{0}}, \mathfrak{g}=\left[\theta \circ \lambda_{*} \circ \mu_{*}\right]$. Then we obtain the family $\pi_{\mathrm{x} i \mathrm{i}}: \mathscr{S} \rightarrow \Delta_{1 / 2} \times \Delta_{0}$, together with sections $\mathfrak{o}$ and $q$ in the same way as in $(2-x)$. Let $\mathfrak{p}$ be the quasi-section of $\pi_{x i 1}$ defined in the same way as in (2-ix). Then $\pi_{\mathrm{x} i \mathrm{i}}^{-1}(0,0)$ is isomorphic to $\pi_{\mathrm{x}}^{-1}(0,0)$ in $(2-\mathrm{x})$, and $\mathfrak{p}(0,0)$ is the pair of the points obtained by identifing the points $(0,1)$ and $(0,-1)$ of $V_{3}$ with the points $(\infty, 1)$ and $(\infty,-1)$ of itself, respectively.

(2-xiii) Over $\Delta_{\infty} \times \tilde{\Delta}_{1 / 2}$.

We can obtain the family $\pi_{\mathrm{xii}}$ : $\mathscr{S} \rightarrow \Delta_{\infty} \times \widetilde{\Delta}_{1 / 2}$, together with the section $\mathfrak{D}$ and the quasi-section $\mathfrak{p}$ of $\pi_{x i 1 i}$ in the same way as in (2-vii). Let $q$ be the section of $\pi_{x i i 1}$ defined in the same way as in (1-xiii). Then clearly $\pi_{\mathrm{xiii}}^{-1}(0,0)(\mathrm{p}(0,0), \mathfrak{p}(0,0))$ is isomorphic to $\pi_{\mathrm{vii}}^{-1}(0,0)(\mathrm{p}(0,0), \mathfrak{p}(0,0))$, and $\mathrm{q}(0,0)$ is the point $\left[1, \sigma_{4}\right]$ of $V_{2}$.

(2-xiv) Over $\Delta_{0} \times \widetilde{\Delta}_{1 / 2}$.

We can obtain the family $\pi_{\mathrm{xiv}}: \mathscr{S} \rightarrow \Delta_{0} \times \tilde{\Delta}_{1 / 2}$, together with the 
section $\mathfrak{p}$ and the quasi-section $\mathfrak{p}$ of $\pi_{\mathbf{x} \mid v}$ in the same way as in (2-viii). Let $q$ be the section of $\pi_{x i v}$ defined in the same way as in (1-xiv). Then clearly $\pi_{\mathrm{xiv}}^{-1}(0,0)(\mathfrak{p}(0,0), \mathfrak{p}(0,0))$ is isomorphic to $\pi_{\mathrm{viii}}^{-1}(0,0)(\mathfrak{p}(0,0), \mathfrak{p}(0,0))$, and $\mathfrak{q}(0,0)$ is the point $\left[1, \sigma_{4}\right]$ of $V_{2}$.

(2-xv) Over $\Delta_{1 / 2} \times \widetilde{\Delta}_{1 / 2}$.

We can obtain the family $\pi_{\mathrm{x} v}: \mathscr{S} \rightarrow \Delta_{1 / 2} \times \tilde{\Delta}_{1 / 2}$, together with the section $\mathfrak{v}$ and the quasi-section $\mathfrak{p}$ of $\pi_{\mathrm{x} v}$ in the same way as in (2-ix). Let $\mathfrak{q}$ be the section of $\pi_{\mathrm{xv}}$ defined in the same way as in (1-xv). Then clearly $\pi_{\mathrm{xv}}^{-1}(0,0)(\mathfrak{p}(0,0),(0,0))$ is isomorphic to $\pi_{\mathrm{ix}}^{-1}(0,0)(\mathfrak{p}(0,0), \mathfrak{p}(0,0))$, and $\mathfrak{q}(0,0)$ is the point $\left[1,-\sigma_{4}\right]$ of $V_{2}$.

(3-i) Over $\Delta_{\infty}$.

Let $\mathscr{A}=\mathscr{E}_{1} \times E\left(\sigma_{3}\right)$, and let $\mathrm{g}:(p,[y]) \mapsto\left(\alpha_{3} p,\left[\sigma_{3} y\right]\right)$ be the automorphism of $\mathscr{A}$. Then $\mathrm{g}$ has three fixed points, and the singular points of $\mathscr{A} / \mathrm{g}^{z}$ are isomorphic to $N_{3}$ of (II) in Section 2. Thus we can obtain the family $\pi_{\mathrm{i}}: \mathscr{S} \rightarrow \Delta_{\infty}$. Let $\mathfrak{p}, \mathfrak{p}$ and $\mathfrak{q}$ be the sections of $\pi_{\mathrm{i}}$ which map $s \in \Delta_{\infty}$ to $[1, s, 0],\left[\sigma_{6}, s, 0\right]$ and $[1, s, 1 / 2] \in \mathscr{S}$, respectively. Then $\pi_{\mathrm{i}}^{-1}(0)$ consists of three components $V_{1,1}, V_{2,1}, V_{3,1}$ isomorphic to $\Sigma_{2}$, three components $V_{1,2}, V_{2,2}, V_{3,2}$ isomorphic to $\boldsymbol{P}^{2}$, and a component $V$ isomorphic to a non-singular model of $\boldsymbol{P}^{1} \times E\left(\sigma_{3}\right) / \bar{g}^{z}$, with $\bar{g}:(\eta,[y]) \mapsto\left(\sigma_{3} \eta,\left[\sigma_{3} y\right]\right)$. Thus $V$ is an elliptic surface over $\boldsymbol{P}^{1}$ with two singular fibers of types IV* and IV. These components intersect as in Figure 3-i. In particular, the points $[0, y]$ of $V$ meet the point $[\infty, y]$ of itself. $\mathfrak{D}(0), \mathfrak{p}(0)$ and $\mathfrak{q}(0)$ are the points $[1,0],\left[\sigma_{\theta}, 0\right]$ and $[1,1 / 2]$ of $V$, respectively.
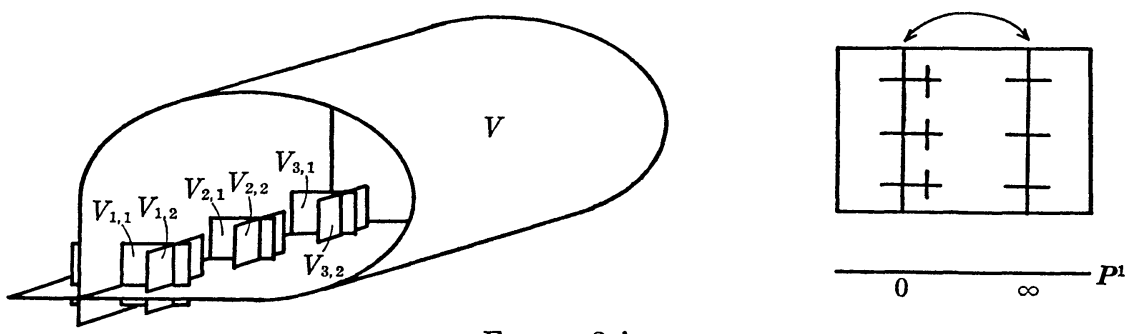

FIGURE 3-i

(3-ii) Over $\Delta_{0}$.

Let $\mathscr{S}=\mathscr{A} / \mathrm{g}^{Z}$, where $\mathscr{A}=\mathscr{E}_{6} \times E\left(\sigma_{3}\right), \mathfrak{g}:(p,[y]) \mapsto\left(\beta^{2} p,\left[\sigma_{3} y\right]\right)$. Then $\mathscr{S}$ is non-singular. Let $\pi_{\mathrm{ii}}: \mathscr{S} \rightarrow \Delta_{0}$ be the holomorphic map induced by $\phi_{6}$. Let $\mathfrak{D}, \mathfrak{p}$ and $\mathfrak{q}$ be the sections of $\pi_{\mathrm{ii}}$ which map $s \in \Delta_{0}$ to $[1, s, 0]$, $[s, s, 0]$ and $[1, s, 1 / 2] \in \mathscr{S}$, respectively. Then $\pi_{\mathrm{ii}}^{-1}(0)$ consists of two components $V_{1}$ and $V_{2}$ both isomorphic to $\boldsymbol{P}^{1} \times E\left(\sigma_{3}\right)$. The points $(0,[y])$ and $(\infty,[y])$ of $V_{1}$ meet the points $\left(\infty,\left[\sigma_{3} y\right]\right)$ and $(0,[y])$ of $V_{2}$, respectively. (See Figure 1-ii.) $\mathfrak{D}(0), \mathfrak{q}(0)$ are the points $(1,[0]),(1,[1 / 2])$ of $V_{1}$ 
respectively, and $\mathfrak{p}(0)$ is the point $(1,[0])$ of $V_{2}$.

(3-iii) Over $\Delta_{1 / 2}$.

Let $\mathscr{S}=\mathscr{A} / \mathfrak{g}^{z}$, where $\mathscr{A}=\mathscr{E}_{3} \times E\left(\sigma_{3}\right), \mathfrak{g}:(p,[y]) \mapsto\left(\alpha_{3} \beta^{2} p,\left[\sigma_{3} y\right]\right)$, and let $\pi_{i i 1}: \mathscr{S} \rightarrow \Delta_{1 / 2}$ be the holomorphic map induced by $\phi_{3}$. Let $\mathfrak{o}, \mathfrak{p}$ and $\mathfrak{q}$ be the sections of $\pi_{\mathrm{ii}}$ which map $s \in \Delta_{1 / 2}$ to $[1, s, 0],\left[\sigma_{6} s, s, 0\right]$ and $[1, s$, $1 / 2] \in \mathscr{S}$, respectively. Then $\pi_{\mathrm{iii}}^{-1}(0)$ consists of a component $V$ whose normalization is isomorphic to $\boldsymbol{P}^{1} \times E\left(\sigma_{3}\right)$. The points $(0,[y])$ meet the points $\left(\infty,\left[\sigma_{3} y\right]\right)$. And $\mathfrak{o}(0), \mathfrak{p}(0)$ and $\mathfrak{q}(0)$ are the points $(1,[0]),(-1,[0])$ and $(1,[1 / 2])$ of $V$, respectively.
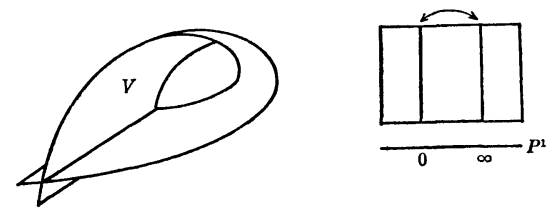

Figure 3-iii

(3-iv) Over $\Delta_{1 / 3}$.

Let $\mathscr{A}=\mathscr{E}_{2} \times E\left(\sigma_{3}\right)$, and let $\mathfrak{g}$ be the automorphism of $\mathscr{A}$ defined in the same way as in (3-i). Then we obtain the family $\pi_{\text {iv }}: \mathscr{S} \rightarrow \Delta_{1 / 3}$ together with sections $\mathfrak{D}$ and $\mathfrak{q}$ in the same way as in (3-i). Let $\mathfrak{p}$ be the section of $\pi_{\mathrm{iv}}$ which maps $s \in \Delta_{1 / 3}$ to $\left[\sigma_{6} s^{3 / 2}, s, 0\right] \in \mathscr{S}$. Then $\pi_{\mathrm{iv}}^{-1}(0)$ is the unramified double covering space of $\pi_{i}^{-1}(0)$ in $(3-i), o(0), q(0)$ are the points [1,0], [1,1/2] of $V^{\prime}$, respectively, and $\mathfrak{p}(0)$ is a point of $V_{1,2}^{\prime}$, where $V^{\prime}$ and $V_{1,2}^{\prime}$ are copies of $V$ and $V_{1,2}$ of $\pi_{i}^{-1}(0)$, respectively.

(4-i) Over $\Delta_{\infty}$.

Let $\mathscr{C}=\mathscr{E}_{3} \times E\left(\sigma_{3}\right) / h^{z}$, where $h:(p,[y]) \mapsto\left(\beta p,\left[y+\left(1-\sigma_{3}\right) / 3\right]\right) . \quad W e$ obtain the family $\pi_{\mathrm{i}}: \mathscr{S} \rightarrow \Delta_{\infty}$ together with the sections $\mathfrak{v}$ and $\mathfrak{q}$ in the same way as in $(3-i)$. Let $\mathfrak{p}$ be the quasi-section of $\pi_{i}$ which maps $s \in \Delta_{\infty}$ to $\left\{\left[\sigma_{6}, s, k\left(1-\sigma_{3}\right) / 3\right] \mid k=0,1,2\right\} \subset \mathscr{S}$. Then $\pi_{1}^{-1}(0)$ consists of the components isomorphic to those in (3-i). But the points $[0, y]$ of $V$ meet the points $\left[\infty, y+\left(1-\sigma_{3}\right) / 3\right] . \mathfrak{p}(0)$ is the triple of the points $\left[\sigma_{6}\right.$, $\left.k\left(1-\sigma_{3}\right) / 3\right], k=0,1,2$, of $V$.

(4-ii) Over $\Delta_{0}$.

Let $\mathscr{A}=\mathscr{E}_{3} \times E\left(\sigma_{3}\right) / h^{z}, \quad$ where $h:(p,[y]) \mapsto\left(\alpha_{3} p,\left[y+\left(1-\sigma_{3}\right) / 3\right]\right)$, and let $\mathrm{g}:[p, y] \mapsto\left[\beta p, \sigma_{3} y\right]$ be the automorphism of $\mathscr{L}$. Then we obtain the family $\pi_{\mathrm{i} i}: \mathscr{S} \rightarrow \Delta_{0}$, together with sections $\mathfrak{D}$ and $\mathfrak{q}$, in the same way as in (3-ii). Let $\mathfrak{p}$ be the quasi-section of $\pi_{\mathrm{ii}}$ which maps $s \in \Delta_{0}$ to $\left\{\left[\sigma_{3}^{k} s^{1 / 3}, s, 0\right] \mid k=0,1,2\right\} \subset \mathscr{S}$. Then $\pi_{\mathrm{ii}}^{-1}(0)$ consists of the component $V$ whose normalization is isomorphic to $\boldsymbol{P}^{1} \times E\left(\sigma_{3}\right) / \bar{h}^{z}$, with $\bar{h}:(\eta,[y]) \mapsto$ $\left(\sigma_{3} \eta,\left[y+\left(1-\sigma_{3}\right) / 3\right]\right)$. Thus $V$ is an elliptic surface over $\boldsymbol{P}^{1}$ with two 

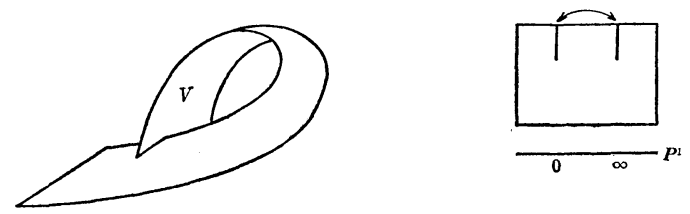

FIGURE 4-ii

triple fibers. The points $[0, y]$ meet the points $\left[\infty, \sigma_{3} y\right]$. And $\mathfrak{o}(0), \mathfrak{q}(0)$ and $\mathfrak{p}(0)$ are the points $[1,0],[1,1 / 2]$ and a point on the double curve of $V$, respectively.

(4-iii) Over $\Delta_{1 / 3}$.

Let $\mathscr{S}$ be the same manifold as in (4-i), and let $\pi_{\mathrm{iii}}: \mathscr{S} \rightarrow \Delta_{1 / 3}$ be the holomorphic map induced by $\dot{\phi}_{3}$. Let $\mathfrak{p}, \mathfrak{q}$ and $\mathfrak{p}$ be the sections and quasi-section of $\pi_{\mathrm{iii}}$ which maps $s \in \Delta_{1 / 3}$ to $[1, s, \delta],[1, s, 1 / 2+\delta] \in \mathscr{S}$ and $\left\{\left[\sigma_{9} s^{\delta}, s, k\left(1-\sigma_{3}\right) / 3+\delta\right] \mid k=0,1,2\right\} \subset \mathscr{S}$, respectively, where $\delta=1 / 3$. Then $\mathfrak{p}(0), \mathfrak{q}(0)$ and $\mathfrak{p}(0)$ are the points $[1 / 3],[1,5 / 6]$ of $V$, and a point on the double curve of $V$, respectively.

(4-iv) Over $\Delta_{-1 / 3}$.

If we set $\delta=-1 / 3$, we obtain the family $\pi_{\mathrm{iv}}: \mathscr{S} \rightarrow \Delta_{-1 / 3}$ together with the sections $\mathfrak{b}, \mathfrak{q}$ and the quasi-section $\mathfrak{p}$, in the same way as in (4-iii).

(5-i) Over $\Delta_{\infty}$.

Let $\mathscr{A}=\mathscr{E}_{1} \times E\left(\sigma_{4}\right)$, and let $\mathrm{g}:(p,[y]) \mapsto\left(\alpha_{4} p,\left[\sigma_{4} y\right]\right)$ be the automorphism of $\mathscr{A}$. Then $\mathscr{A} / \mathrm{g}^{Z}$ has two singular points isomorphic to $N_{2}$, and a singular point isomorphic to $N_{4}$. Thus we obtain the family $\pi_{\mathrm{i}}: \mathscr{S} \rightarrow \Delta_{\infty}$. Let $\mathfrak{D}$ and $\mathfrak{q}$ be the sections of $\pi_{\mathrm{i}}$, which map $s \in \Delta_{\infty}$ to $[1, s, 0]$ and $[1, s, 1 / 4] \in \mathscr{S}$, respectively. Then $\pi_{1}^{-1}(0)$ consists of two components $V_{1,1}, V_{2,1}$ isomorphic to $\Sigma_{3}$, two components $V_{1,2}, V_{2,2}$ isomorphic to $\Sigma_{2}$, three components $V_{1,3}, V_{2,3}, V_{3}$ isomorphic to $\boldsymbol{P}^{2}$, and a component $V$ isomorphic to a non-singular model of $\boldsymbol{P}^{1} \times E\left(\sigma_{4}\right) / \bar{g}^{z}$, where $\bar{g}:(\eta,[y]) \mapsto\left(\sigma_{4} \eta,\left[\sigma_{4} y\right]\right)$. Thus $V$ is an elliptic surface over $\boldsymbol{P}^{1}$ with two singular fibers of types III* and III. These components intersect as in
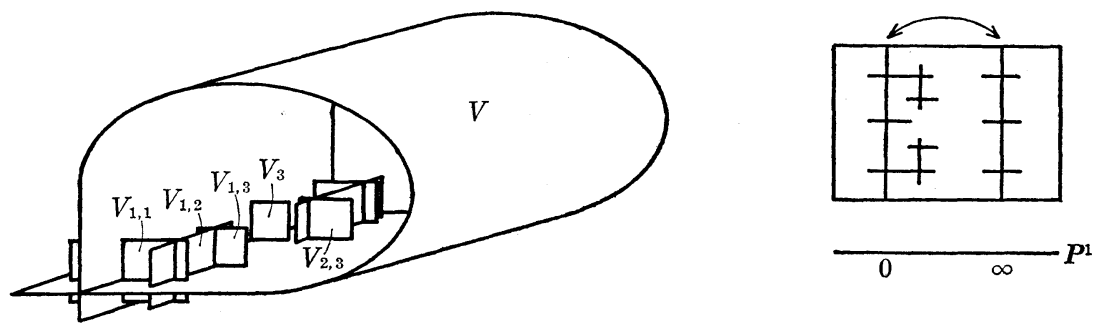

FIGURE 5-i 
Figure 5-i. In particular, the points $[0, y]$ of $V$ meet the points $[\infty$, $\left.\sigma_{4} y\right]$ of itself. And $\mathrm{D}(0)$ and $\mathfrak{q}(0)$ are the points $[1,0]$ and $[1,1 / 4]$ of $V$, respectively.

(5-ii) Over $\Delta_{0}$.

Let $\mathscr{S}=\mathscr{A} / \mathrm{g}^{z}$, where $\mathscr{A}=\mathscr{C}_{4} \times E\left(\sigma_{4}\right), \mathrm{g}:(p,[y]) \mapsto\left(\beta p,\left[\sigma_{4} y\right]\right)$. Let $\pi_{\mathrm{ii}}: \mathscr{S} \rightarrow \Delta_{0}$ be the holomorphic map induced by $\phi_{4}$, and let $\mathfrak{o}$ and $\mathfrak{q}$ be the sections of $\pi_{\mathrm{ii}}$ defined in the same way as in (5-i). Then $\pi_{\mathrm{ii}}^{-1}(0)$ consists of a component $V$ whose normalization is isomorphic to $P^{1} \times E\left(\sigma_{4}\right)$. The points $(0,[y])$ meet the points $\left(\infty,\left[\sigma_{4} y\right]\right)$. And $\mathfrak{D}(0), \mathfrak{q}(0)$ are the points $(1,[0]),(1,[1 / 4])$ of $V$, respectively. (See Figure 3-iii.)

(5-iii) Over $\Delta_{1 / 2}$.

Let $\mathscr{A}=\mathscr{E}_{2} \times E\left(\sigma_{4}\right)$, and let $\mathrm{g}:(p,[y]) \mapsto\left(\alpha_{4} \beta p,\left[\sigma_{4} y\right]\right)$ be the automorphism of $\mathscr{A}$. Then $\mathscr{A} / \mathrm{g}^{z}$ has four singular points isomorphic to $N_{2}$. Thus we obtain the family $\pi_{i i 1}: \mathscr{S} \rightarrow \Delta_{1 / 2}$ together with sections $\mathfrak{o}$ and $q$ defined in the same way as in $(5-i)$. Then $\pi_{i i i}^{-1}(0)$ consists of five components isomorphic to those of $\pi_{\mathrm{i}}^{-1}\left(0,\left[\sigma_{4}\right]\right)$ in (1-i). But the points $[\infty, y]$ of $V$ meet the points $\left[0, \sigma_{4} y\right]$ of itself.

(6-i) Over $\Delta_{\infty}$.

Let $\mathscr{A}=\mathscr{E}_{2} \times E\left(\sigma_{4}\right) / h^{Z}$, where $h:(p,[y]) \mapsto\left(\beta p,\left[y+\left(1+\sigma_{4}\right) / 2\right]\right)$. Then we obtain the family $\pi_{\mathrm{i}}$ : $\mathscr{S} \rightarrow \Delta_{\infty}$, together with sections $\mathfrak{D}$ and $\mathfrak{q}$ in the same way as in (5-i). Then $\pi_{i}^{-1}(0)$ consists of eight components isomorphic to those of $\pi_{i}^{-1}(0)$ in $(5-i)$. (See Figure 5-i). In particular, the points $[\infty, y]$ of $V$ meet the points $\left[0, y+\left(1+\sigma_{4}\right) / 2\right]$ of itself.

(6-ii) Over $\Delta_{0}$.

Let $\mathscr{L}=\mathscr{E}_{4} \times E\left(\sigma_{4}\right) / h^{Z}$, where $h:(p,[y]) \mapsto\left(\alpha_{2} p,\left[y+\left(1+\sigma_{4}\right) / 2\right]\right)$, and let $g$ be the automorphism of $\mathscr{A}$ defined in the same way as in (5-ii). Then we obtain the family $\pi_{\mathrm{i} i}: \mathscr{S} \rightarrow \Delta_{0}$, together with the sections 0 and $\mathfrak{q}$ in the same way as in (5-ii). Then $\pi_{\mathrm{ii}}^{-1}(0)$ consists of the component $V$ isomorphic to $\boldsymbol{P}^{1} \times E\left(\sigma_{4}\right) / \bar{h}^{z}$, where $\bar{h}:(\eta,[y]) \mapsto\left(-\eta,\left[y+\left(1+\sigma_{4}\right) / 2\right]\right)$, with the points $[0, y]$ meeting the points $\left[\infty, \sigma_{4} y\right]$. (See Figure 2-ii.)

(6-iii) Over $\Delta_{1 / 2}$.

Let $\mathscr{A}=\mathscr{E}_{4} \times E\left(\sigma_{4}\right) / h^{z}$, where $h:(p,[y]) \mapsto\left(\beta^{2} p,\left[y+\left(1+\sigma_{4}\right) 2\right]\right)$. Then we obtain the family $\pi_{\mathrm{iii}}: \mathscr{S} \rightarrow \Delta_{1 / 2}$, together with sections 0 and $q$ in the same way as in (5-iii). Then $\pi_{i i 1}^{-1}(0)$ consists of the five components isomorphic to those of $\pi_{\mathrm{iii}}^{-1}(0)$ in (5-iii). But the points $\left(\left[0, y+\left(1+\sigma_{4}\right) / 2\right]\right)$ of $V$ meet the points $[\infty, y]$ of itself $\mathfrak{o}(0)$ and $\mathfrak{q}(0)$ are the points $[1,1 / 2]$ and $[1,3 / 4]$, respectively. (See Figure 1-i.)

(6-iv) Over $\Delta_{1 / 4}$. 
Let $\mathrm{g}:[p, y] \mapsto\left[\alpha_{4} \beta p, \sigma_{4} y\right]$ be the automorphism of $\mathscr{A}$, where $\mathscr{A}$ is the same manifold as in (6-i). Then we obtain the family $\pi_{\mathrm{iv}}: \mathscr{S} \rightarrow \Delta_{1 / 4}$ together with sections $\mathfrak{D}$ and $q$ in the same way as in (6-i). Then $\pi_{\mathrm{iv}}^{-1}(0)$ is isomorphic to $\pi_{1}^{-1}(0)$ in $(6-\mathrm{i}), \mathfrak{o}(0)$ and $\mathfrak{q}(0)$ are the points $[1,1 / 2]$ and $\left[1, \sigma_{4} / 2\right]$ of $V$, respectively.

\section{(7-i) Over $\Delta_{\infty}$.}

Let $\mathscr{A}=\mathscr{O}_{1} \times E\left(\sigma_{3}\right)$, and let $\mathfrak{g}:(p,[y]) \mapsto\left(\alpha_{6} p,\left[-\sigma_{3} y\right]\right)$ be the automorphism of $\mathscr{A}$. Then $\mathscr{A} / \mathrm{g}^{z}$ has three singular points isomorphic to $N_{6}, N_{3}$ and $N_{2}$, respectively. Thus we obtain the family $\pi_{\mathrm{i}}: \mathscr{S} \rightarrow \Delta_{\infty}$. Let $\mathfrak{v}$ and $\mathfrak{q}$ be the sections of $\pi_{\mathrm{i}}$, which map $s \in \Delta_{\infty}$ to $[1, s, 0]$ and [1, $s, 1 / 3]$, respectively. Then $\pi_{1}^{-1}(0)$ consists of components $V_{1,1}, V_{1,2}, V_{1,3}$, $V_{1,4}, V_{1,5}, V_{2,1}, V_{2,2}, V_{3}$ isomorphic to $\Sigma_{5}, \Sigma_{4}, \Sigma_{3}, \Sigma_{2}, \boldsymbol{P}^{2}, \Sigma_{2}, \boldsymbol{P}^{2}, \boldsymbol{P}^{2}$, respectively, and a component $V$ isomorphic to a non-singular model of $\boldsymbol{P}^{1} \times E\left(\sigma_{3}\right) / \bar{g}^{z}$, where $\bar{g}:(\eta,[y]) \mapsto\left(\sigma_{6} \eta,\left[-\sigma_{3} y\right]\right)$. Thus $V$ is an elliptic surface over $\boldsymbol{P}^{1}$ with two singular fibers of types II* and II. These components intersect as in Figure (7-i). In particular, the points $[0, y]$ of $V$ meet the points $[\infty, y]$ of itself. $\mathfrak{d}(0)$ and $\mathfrak{q}(0)$ are the points $[1,0]$ and $[1,1 / 3]$ of $V$, respectively.
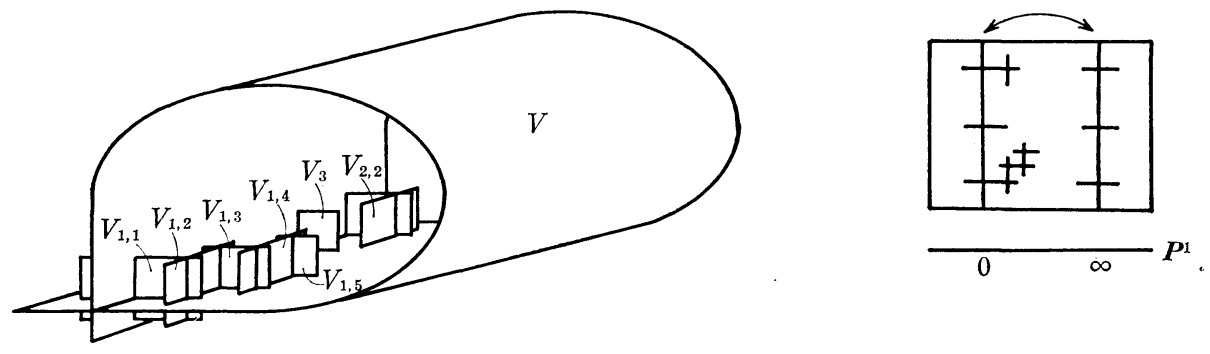

FiguRE 7-i

(7-ii) Over $\Delta_{0}$.

Let $\mathscr{S}=\mathscr{A} / \mathrm{g}^{Z}$, where $\mathscr{A}=\mathscr{E}_{6} \times E\left(\sigma_{3}\right), \mathfrak{g}:(p,[y]) \mapsto\left(\beta^{-1} p,\left[-\sigma_{3} y\right]\right)$. Let $\pi_{\mathrm{ii}}: \mathscr{S} \rightarrow \Delta_{0}$ be the holomorphic map induced by $\phi_{6}$, and let $\mathfrak{D}$ and $\mathrm{q}$ be the sections of $\pi_{\mathrm{ii}}$ defined in the same way as in (7-i). Then $\pi_{\mathrm{ii}}^{-1}(0)$ consists of the component $V$ isomorphic to $\boldsymbol{P}^{1} \times E\left(\sigma_{3}\right)$. The points $(0,[y])$ meet the points $\left(\infty,\left[-\sigma_{3} y\right]\right)$.

(7-iii) Over $\Delta_{1 / 2}$.

Let $\mathscr{A}=\mathscr{E}_{3} \times E\left(\sigma_{3}\right)$, and let $\mathfrak{g}:(p,[y]) \mapsto\left(\alpha_{6} \beta p,\left[-\sigma_{3} y\right]\right)$ be the automorphism of $\mathscr{A}$. Then $\mathscr{A} / \mathrm{g}^{z}$ has four singular points isomorphic to $N_{2}$. Thus we obtain the family $\pi_{i 11}: \mathscr{S} \rightarrow \Delta_{1 / 2}$, together with the sections $\mathrm{v}$ and $\mathfrak{q}$ defined in the same way as in $(7-\mathrm{i})$. Then $\pi_{\mathrm{iii}}^{-1}(0)$ consists of five com- 
ponents isomorphic to those of $\pi_{\mathrm{i}}^{-1}\left(0,\left[\sigma_{3}\right]\right)$ in (1-i). But the points $[\infty, y]$ of $V$ meet the points $\left[0,-\sigma_{3} y\right]$ of itself.

(7-iv) Over $\Delta_{1 / 3}$.

Let $\mathscr{A}=\mathscr{E}_{2} \times E\left(\sigma_{3}\right)$, and let $\mathfrak{g}$ be the automorphism of $\mathscr{A}$ defined in the same way as in (7-iii). Then $\mathscr{A} / \mathrm{g}^{z}$ has three singular points isomorphic to $N_{3}$. Thus we obtain the family $\pi_{\mathrm{i} v}$ : $\mathscr{S} \rightarrow \Delta_{1 / 3}$, together with the sections $\mathfrak{D}$ and $q$ defined in the same way as in (7-i). Then $\pi_{\mathrm{iv}}^{-1}(0)$ consists of seven components isomorphic to those of $\pi_{i}^{-1}(0)$ in (3-i). But the points $[\infty, y]$ of $V$ meet the points $\left[0,-\sigma_{3} y\right]$ of itself.

\section{REFERENCES}

[1] F. Enriques et F. Severi, Mémoire sur les surfaces hyperelliptique, Acta Math., 32 (1909), 283-392, 33 (1910), 321-403.

[2] R. C. Gunning, Lectures on Riemann Surfaces, Princeton Univ. Press, 1965.

[3] G. KempF ET AL., Toridal Embeddings, I, Lecture Notes in Math. 339, Springer-Verlag, Berlin-Heidelberg-New York, 1973.

[4] K. Kodaira, On compact analytic surfaces II, Ann. Math., 77 (1963), 563-626.

[5] T. ODA, Lectures on torus embeddings and applications (Based on joint work with $\mathrm{K}$. Miyake), Tata Inst. of Fund. Res., 1978.

[6] D. Mumford, Abelian Varieties, Oxford Univ. Press, 1970.

[7] D. MUMFoRd, A new approach to compactifing locally symmetric varieties, in Discrete subgroups of Lie groups, Oxford Univ. Press, 1975.

[8] D. MumfoRd, Geometric Invariant Theory, Springer-Verlag, Berlin-Heidelberg-New York, 1965.

[9] D. MUmford AND K. Suominen, Introduction to the theory of moduli, in Algebraic Geometry, Oslo, 1970.

[10] U. Persson, On degenerations of algebraic surfaces, Memoire of Amer. Math. Soc., 189 (1977).

[11] T. SuwA, On hyperelliptic surfaces, J. Fac. Sci. Univ. Tokyo Sec. I, 14 (1970), 469-476.

[12] K. UENo, On fiber spaces of normally polarized abelian varieties of dimensions 2, I, J. Fac. Sci. Univ. Tokyo, Sec. I. A, 17 (1971), 37-95.

Mathematical Institute

TôHOKU UNIVERSITY

SENDAI, 980 JAPAN 
